\title{
COORDINATIZATION APPLIED TO FINITE BAER * RINGS
}

\author{
BY \\ DAVID HANDELMAN(')
}

\begin{abstract}
We clarify and algebraicize the construction of the 'regular rings' of finite Baer * rings. We first determine necessary and sufficient conditions of a finite Baer * ring so that its maximal ring of right quotients is the 'regular ring', coordinatizing the projection lattice. This is applied to yield significant improvements on previously known results: If $R$ is a finite Baer * ring with right projections *-equivalent to left projections ( $L P \sim R P$ ), and is either of type II or has 4 or more equivalent orthogonal projections adding to 1 , then all matrix rings over $R$ are finite Baer * rings, and they also satisfy $L P \sim R P ;$ if $R$ is a real $A W^{*}$ algebra without central abelian projections, then all matrix rings over $R$ are also $A W^{*}$.

An alternate approach to the construction of the 'regular ring' is via the Coordinatization Theorem of von Neumann. This is discussed, and it is shown that if a Baer * ring without central abelian projections has a 'regular ring', the 'regular ring' must be the maximal ring of quotients. The following result comes out of this approach: A finite Baer * ring satisfying the 'square root' (SR) axiom, and either of type II or possessing 4 or more equivalent projections as above, satisfies $L P \sim R P$, and so the results above apply.

We employ some recent results of J. Lambek on epimorphisms of rings. Some incidental theorems about the existence of faithful epimorphic regular extensions of semihereditary rings also come out.
\end{abstract}

This work arose from two sources: frequent profitable discussions with Professor J. Lambek, and the comment on p. 212 of [2], which asks if there is any connection between a Baer * ring whose lattice of projections is modular and the regular ring coordinatizing the lattice (the answer is Yes!).

It is with the greatest pleasure that I acknowledge my debt to Professor J. Lambek, my advisor. Letters and preprints from Professor S. K. Berberian, and an interesting conversation with Professor G. Michler helped me clarify many ideas and results.

The terminology is that of [13] and [14]. It is assumed the reader has some familiarity with all of the following: Baer * rings [13], the maximal or complete ring of quotients [14, p. 94 on ], (von Neumann) regular rings, and flat epimorphisms of rings [21].

Received by the editors November 10, 1975 and, in revised form, March 12, 1976.

(')Supported by a Postdoctoral Fellowship from the National Research Council of Canada.

AMS (MOS) subject classifications (1970). Primary 46K99; Secondary 06A30, 06A25, 16A08, 16A28, 16A34, 16A52, 16A80, 46L10.

Key words and phrases. Finite Baer * ring, epimorphism of ring, maximal ring of quotients, complete *-regular ring, complemented modular lattice, continuous geometry. 
If $R$ is a ring, we mean it to be associative and possess 1; the symbol $M_{n} R$ denotes the ring of $n$ by $n$ matrices over $R$. A ring $R$ is said to have order $n$ for some integer $n$ if there is a ring $S$ such that $R$ is isomorphic to $M_{n} S$.

A complemented modular lattice will have its biggest element denoted by 1 and its smallest by 0 . The inf and sup operations will be denoted by $\Lambda$ and $\bigvee$, respectively. We will say that a complemented modular lattice has order $n$ if there is a collection of $n$ pairwise perspective, independent elements whose supremum is 1 in the lattice.

If $t$ is an element of a ring $R$, we shall denote the set $\{r \in R: t r=0\}$ (the right annihilator of $t$ ) by $t^{r}$ or $t^{r_{R}}$ if there is ambiguity concerning the ring in which the annihilator is being taken. The left annihilator will be denoted $t^{l}$ or $t^{t_{R}}$. This notation will also be used to denote the annihilators of sets.

We require a number of preliminary results of a purely ring-theoretic nature.

1. Intimate extensions. A ring homomorphism $\beta: R \rightarrow S$ is called intimate (on the right) if $\beta$ is one-to-one and every principal right ideal of $S$ is generated by an element of the image of $R$. We shall call $\beta$ intimate if it is both right and left intimate. We will say an inclusion $R \subset S$ is intimate if the inclusion map is intimate.

The notion of intimacy and many of the results in this section are due to J. Lambek.

A ring inclusion $R \subset S$ is called (left) slightly flat [7, p. 72] if for all $r$ in $R$,

$$
r^{r_{\mathrm{R}}} \cdot S=r^{r_{s}} \text {. }
$$

Equivalently the canonical map $r R \otimes S \rightarrow r S$ is an isomorphism of right $S$-modules.

LEMMA 1.1. Let $R \subset S$ be a right intimate inclusion of rings and suppose $S$ is a regular ring. The $R \subset S$ is left slightly flat.

Proof. There exists $e=e^{2}$ in $S$ such that $r^{r}=e S$. There exists $t$ in $R$ with $e S=t S$. Obviously $t$ belongs to $r^{r_{S}} \cap R=r^{r_{R}}$, so $t S \subset r^{r_{R}} \cdot S$. Trivially, $r^{r_{R}} \cdot S \subset r^{r_{S}}$, so $t S=r^{r_{R}} \cdot S$.

LEMMA 1.2. Suppose $R \subset S$ is an inclusion of rings such that for infinitely many integers $n$, the induced map $M_{n} R \subset M_{n} S$ is slightly flat. Then ${ }_{R} S$ is flat.

Proof. It suffices to show for any finitely generated right ideal $\Sigma r_{i} R$, the natural map $\left(\Sigma r_{i} R\right) \otimes S \rightarrow \Sigma r_{i} S$ is an isomorphism [14, p. 133]. There exists an integer $n$ larger than the number of generators of $\Sigma r_{i} R$, such that $M_{n} R \subset M_{n} S$ is slightly flat. Now $\Sigma r_{i} R$ can be regarded as a principal right ideal of $M_{n} r$ and the result follows.

TheOREM 1.3 (LAMBEK). Let $R$ be a ring, and $\left\{r_{n}\right\}_{n \in Z}$ a subset of $R$. There 
eixsts at most one solution $\left\{x_{n}\right\}_{n \in \mathbf{Z}}$ in $R$ to the collection of equations

$$
r_{n}=r_{n} x_{n} r_{n}, \quad x_{n} r_{n}=r_{n+1} x_{n+1}, \quad x_{n} r_{n} x_{n}=x_{n} \text {. }
$$

Proof. Let $\left\{y_{n}\right\}_{n \in Z}$ be another solution of these equations. We obtain

$$
\begin{aligned}
x_{n} & =x_{n} r_{n} x_{n}=r_{n+1} x_{n+1} x_{n}=\left(r_{n+1} y_{n+1} r_{n+1}\right) x_{n+1} x_{n} \\
& =\left(y_{n} r_{n}\right)\left(x_{n} r_{n}\right) x_{n}=y_{n} r_{n} x_{n}=\left(y_{n} r_{n} y_{n}\right) r_{n} x_{n} \\
& =y_{n}\left(y_{n-1} r_{n-1}\right)\left(x_{n-1} r_{n-1}\right)=y_{n} y_{n-1} r_{n-1} \\
& =y_{n} r_{n} y_{n}=y_{n} . \square
\end{aligned}
$$

A ring homomorphism $R \rightarrow S$ is said to be an epimorphism [15] (in the category of rings) if for any two homomorphisms $f, g: S \rightarrow U$ such that $f=g$ on the image of $R$, we have $f=g$.

TheOREM 1.4 [15, TheOREM 2.3]. Let $K$ be an ideal of $T=R \circ \mathbf{Z}[X]$, the free product of $R$ with the free Z-algebra on a set $X$. Then the polynomials in $K$ have a unique solution in every extension ring of $R$ if and only if $R \rightarrow T / K$ is an epimorphism of rings.

For instance, if $X=\left\{z_{n}\right\}_{n \in Z},\left\{r_{n}\right\} \subset R$, and three classes of polynomials are defined by

$$
\begin{gathered}
f_{n, 1}=r_{n} z_{n} r_{n}-r_{n}, \quad f_{n, 2}=z_{n} r_{n}-r_{n+1} z_{n+1}, \\
f_{n, 3}=z_{n} r_{n} z_{n}-z_{n},
\end{gathered}
$$

we define $K$ to be the two-sided ideal of the ring $T=R \circ \mathbf{Z}\left[z_{n}\right]$, generated by these polynomials. Then the obvious map $R \rightarrow T / K$ is an epimorphism of rings. In general, there is no way to guarantee the map is either one-to-one or onto, or even nonzero.

Proposition 1.5 (LAMBEK). Let $R \subset S$ be an intimate extension of rings, and suppose $S$ is a regular ring. Then there exists an intermediate ring $T$, such that $R \subset T$ is an epimorphism and $T$ is regular.

Proof. Order by inclusion all the rings $S^{\prime}$ with $R \subset S^{\prime} \subset S$ such that the inclusion of $R$ in $S^{\prime}$ is an epimorphism ( $R \subset R$ is trivially an epimorphism). By either Isbell's Zigzag Theorem [10] or abstract nonsense, we see that the union of a chain of epimorphic inclusions is still an epimorphism, so Zorn's Lemma applies. Thus there is an intermediate ring $T$ such that $R \subset T$ is a maximal epimorphic extension of $R$ within $S$. If $T$ were not regular, there would exist $r$ in $T$ such that $r x r=r$ has no solution in $T$. We define a pair of sequences $\left\{r_{n}\right\},\left\{s_{n}\right\}$ in order to apply 1.3 and 1.4.

Clearly $T \subset S$ is intimate. Set $r_{0}=r$. As $S$ is regular there exists $s_{0}$ in $S$ such that $r s_{0} r=r$ and $s_{0} r s_{0}=s_{0}$. Assuming $\left\{r_{n}\right\}_{n=0}^{m},\left\{s_{n}\right\}_{n=0}^{m}$ are defined for some $m>0$ to satisfy 


$$
\begin{aligned}
r_{n} & =r_{n} s_{n} r_{n}, & & 0 \leqslant n \leqslant m, \\
s_{n} r_{n} & =r_{n+1} s_{n+1}, & & 0 \leqslant n \leqslant m-1, \\
s_{n} & =s_{n} r_{n} s_{n}, & & 0 \leqslant n \leqslant m,
\end{aligned}
$$

we define $s_{m+1}$ and $r_{m+1}$ to satisfy the equations of 1.3. Now $s_{m} r_{m}$ is idempotent and as $s_{m} S=r^{\prime} S$ for $r^{\prime}$ in $T$, there exists $s^{\prime}$ such that $s_{m} r_{m}=r^{\prime} s^{\prime}$. Set $r_{m+1}=r^{\prime}$ and $s_{m+1}=s^{\prime} r^{\prime} s^{\prime}$. As $s_{m} S=s_{m} r_{m} S=r^{\prime} S$ and $s_{m} r_{m}=r^{\prime} s^{\prime}$ is an idempotent,

$$
r_{m+1}=r^{\prime}=s_{m} r_{m} r^{\prime}=r^{\prime} s^{\prime} r^{\prime}=r^{\prime} s^{\prime} r^{\prime} s^{\prime} r^{\prime}=r_{m+1} s_{m+1} r_{m+1}
$$

similarly, as $\left(s^{\prime} r^{\prime}\right)^{3}=\left(s^{\prime} r^{\prime}\right)^{2}, s_{m+1}=s_{m+1} r_{m+1} s_{m+1}$. Finally,

$$
r_{m+1} s_{m+1}=r^{\prime} s^{\prime} r^{\prime} s^{\prime} r^{\prime} s^{\prime}=\left(r^{\prime} s^{\prime}\right)^{3}=r^{\prime} s^{\prime}=s_{m} r_{m} .
$$

Analogously, $r_{-n}, s_{-n}$ can be defined in the same way.

Form the free product $U=T \circ \mathbf{Z}\left[z_{n}\right]_{n \in Z}$, and let $K$ be the two-sided ideal generated by the polynomials $f_{n, i}$ defined following Theorem 1.4. There is a map $U \rightarrow S$ given by $t \mapsto t, z_{n} \mapsto S_{n}$. Let $V$ be the image of this map. Then the diagram

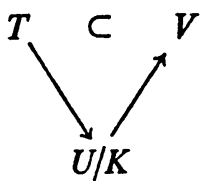

commutes, $T \rightarrow U / K$ is an epimorphism of rings by 1.4 and 1.3 , and $U / K \rightarrow V$ is an onto map. Thus $T \subset V$ is a composition of epimorphism, so is an epimorphism. By the maximality of $T$, we must have $T=V$; but this contradicts $s_{0}$ not belonging to $T$. Hence, the assumption that $T$ is not regular is not valid.

If $D$ is any domain and $S$ is any division ring containing $D$, then the inclusion is trivially an intimate extension, but is almost never an epimorphism. However, we now show that this example is essentially the only exception to the reasonable conjecture that if $R \subset S$ is intimate and $S$ is regular, then $S$ is an epimorphism of $R$.

LEMMA 1.6 (VON NEUMANN). If $e$ and $f$ are idempotents in a ring $R$, satisfying $e R=f R$ and $(1-e) R=(1-f) R$, then $e=f$.

Proof. From $(1-e)(1-f)=1-f$ we deduce $1-e-f+e f=1-f$, whence $e=e f$. But $e R=f R$, so $f=e f$. Thus $e=e f=f$.

Proposirion 1.7. If $T$ is a regular ring and $T \subset S$ is a right or left intimate extension, then $T$ contains all the idempotents of $S$.

Proof. Choose $f=f^{2}$ in $S$. There exist $t, u$ in $T$ such that $t S=f S$ and 
$u S=(1-f) S$. Now $t T \cap u T \subset t S \cap u S=(0)$, so $t T+u T=t T \oplus u T$. If $t T \oplus u T \neq T$, by the regularity of $T$ there exists nonzero $x$ in $T$ with $x t=x u=0$; this immediately yields a contradiction in $S$. Hence $t T \oplus u T=$ $T$, so there exists an idempotent $e$ in $T$ with $e T=t T$ and $(1-e) T=u T$. Thus $e S=f S$ and $(1-e) S=(1-f) S$, so by the preceding lemma, $e=f$.

COROLlary 1.8. Suppose $T$ is regular, $T \subset S$ is a right or left intimate extension, and either of the following conditions hold:

(a) $S$ is simple, regular, but not a division ring,

(b) $S$ is an $n \times n$ matrix ring over some other ring for $n \geqslant 2$. Then $T=S$.

Proof. In either case $S$ is generated by its idempotents.

THEOREM 1.9. Let $R \subset S$ be an intimate extension of rings, with $S$ regular. Suppose any of the following conditions hold:

(a) $S$ is simple but not a division ring,

(b) $R$ is an $n \times n$ matrix ring over another ring for some $n \geqslant 2$,

(c) $S$ is an $n \times n$ matrix ring over another ring for some $n \geqslant 2$,

(d) $S$ is generated, as a ring, by its idempotents.

Then $R \subset S$ is an epimorphism of rings.

Proof. It is easy to see that (b) implies (c), which implies (d). Also (a) implies (d), so the result follows directly from 1.5 and 1.7.

COROLlary 1.10. Suppose $R \subset S$, where $S$ is regular and for infinitely many positive integers $n$, the induced map $M_{n} R \subset M_{n} S$ is intimate. Then $R \subset S$ is a flat epimorphism of rings; in particular, $S$ is a ring of quotients of $R$.

Proof. Follows immediately from Lemmas 1.1, 1.2 and Theorem 1.9.

It would result in a considerable simplification if we could show:

If $M_{n} R \subset M_{n} S$ for some $n \neq 1$ is intimate, and $S$ is regular, then $R \subset S$ is a flat epimorphism.

Another result which should be trivial, but which I have not been able to prove:

If $M_{2} R \subset M_{2} S$ is intimate and $S$ is regular, then $R \subset S$ is intimate.

2. Maximal rings of quotients of Baer * rings. A ring $R$ is a Baer * ring [13] if there is an involution * such that for any subset $S$, of $R$, there is an idempotent $p$ with $p=p^{*}$ (called a projection) such that $S^{r}=p R$. This implies there is a projection $q$ such that $S^{l}=R q$. We call $p(q)$ the right (left) annihilating projection of $S$. 
Denote the lattice of right annihilators of subsets of $R$, by $L(1, R)$. This is always complete, and in the case of a Baer * ring, is orthocomplemented. In particular, the collection of projections of $R$ forms a complete lattice, as $(1-p)^{r}=p R$; and we may identify the projection lattice with $L(1, R)$.

If $r$ belongs to $R$, there is a smallest projection $p$ such that $p r=r$ : take the left annihilating projection of $r$, say $q$, then take the right annihilating projection of $q$, which is, of course, $1-q$, so $p=1-q$. We say $p$ is the left projection of $r$, abbreviated to $\operatorname{LP}(r)=p$. The right projection is defined similarly (RP), and we have the formulae:

$$
\begin{aligned}
& \mathrm{LP}(r)=p \text {, where } p \text { is a projection and } p R=r^{l r}\left(=\left(r^{l}\right)^{r}\right) \text {. } \\
& \operatorname{RP}(r)=p^{\prime} \text {, where } p^{\prime} \text { is a projection and } R p^{\prime}=r^{r l} \text {. }
\end{aligned}
$$

Given $p, q$ projections, how may we determine $p \wedge q, p \vee q$ in the lattice? To answer this, we first note that the partial ordering is given by $p<q$ if $p q=p$ (which is equivalent to $p=q p=p q)$.

Proposition 2.1. Let $R$ be a Baer * ring, and suppose $\left\{p_{i}\right\}$ is a collection of projections in $R$. Then

(a) $\sup p_{i}=q$, where $q$ is the projection such that $q R=\left(\Sigma p_{i} R\right)^{l r}$.

(b) inf $p_{i}=p$, where $p$ is the projection such that $p R=\bigcap p_{i} R$.

Proof. (a) As $p_{i}$ belongs to $\left\{p_{i}\right\}^{l r}, p_{i}$ belongs to $q R$, so $q p_{i}=p_{i}$, i.e., $p_{i}<q$. If $x$ is any other projection such that $p_{i}<x$ (for all $i$ ), then $\left\{p_{i}\right\} \subset x R$, so taking left annihilators (inclusion-reversing) and then right annihilators (again inclusion-reversing), we see $q R=\left\{p_{i}\right\}^{l r} \subset x^{l r}=x R$, so $q \leqslant x$. Thus $q=$ $\sup p_{i}$.

(b) Obviously, $\cap p_{i} R=\left\{1-p_{i}\right\}^{r}$, so there exists a projection $p$ such that $p R=\bigcap p_{i} R$. That $p$ is the infimum is clear.

For suitable Baer * rings $R$, Berberian showed there is a complete *-regular ring [12] $C$, such that $R \subset C$ (as a subring), the involution extends, and all the projections of $C$ lie in $R$; so $L(1, C)=L(1, R)$; i.e., $C$ coordinatizes the projection lattice. For finite $\mathrm{Baer}^{*}$ rings satisfying the EP and $\mathrm{SR}$ axioms, Roos [20] claimed to have shown that $C$ is nothing more than the maximal ring of quotients of $R$.

In general, then, if $L(1, R)$ is the projection lattice of $R$, we would like to find a complete regular ring $Q$ and an embedding $R \rightarrow Q$ such that the induced map of lattices of annihilators is an isomorphism, that is, the map is an intimate extension of rings; it turns out that the involution extends automatically. Such a $Q$ we will call the 'regular ring' of $R$. In this section, we determine necessary and sufficient conditions for the maximal ring of quotients to be the 'regular ring'. These conditions are much weaker than those required by Berberian or Roos. In a later section, it will be shown that 
if a 'regular ring' exists, and the Baer * ring has no central abelian projections, then the 'regular ring' must be the maximal ring of quotients.

In $\S \S 3,4$, the results of this section will be used to prove new results on matrix rings over finite Baer * rings.

If $R$ is a Baer * ring and has a 'regular ring', then $L(1, R)$ must be a modular lattice. This motivates (as we shall see) the following definition:

A Baer * ring is strongly modular, if for all $r$ in $R, r^{r}=(0)$ implies $r R$ is an essential right $R$-ideal, that is, $r R$ has nonzero intersection with every nonzero right ideal.

Since a right ideal $E$ is essential as a right ideal $E$ if and only if $E^{*}$ is an essential left ideal, the definition is right-left symmetric.

PROPOSITION 2.2. Let $R$ be a strongly modular Baer * ring. Then

(a) for all $a$ in $R, a R$ is an essential submodule of $a^{\text {lr; }}$;

(b) $L(1, R)$, the projection lattice of $R$, is modular.

Proof. (a) Choose $a$ in $R$, and let $p=\operatorname{LP}(a)$, so $a^{l r}=p R$. We have $a R \subset p R$, so $a R \cap(1-p) R=(0)$. Consider the element $z=a a^{*}+1-p$. Let $q$ be the right annihilating projection of $z$, so $z^{r}=q R$. Then

$$
a a^{*} q+(1-p) q=0
$$

from $a R \cap(1-p) R=(0)$, we obtain $a a^{*} q=(1-p) q=0$. Applying the involution, $q a a^{*}=q(1-p)=0$. Thus $(q a)(q a)^{*}=0$, so $q a=0$, whence $q$ belongs to $a^{l}=a^{l r l}$, which equals $R(1-p)$, so $q=q(1-p)=0$. Thus $z^{r}=(0)$, whence $z R$ is an essential right ideal. If there exists $b$ in $p R$ with $b R \cap a R=(0)$, then

$$
z R \cap b R \subset(a R \oplus(1-p) R) \cap b R=(0),
$$

so $b=0$, verifying the essentiality.

(b) If $L(1, R)$ is not modular, we may find principal right ideals $e R, f R, g R$ with $e, f, g$ projections such that $e R \subset f R, g R \cap f R=(0)$, and $(e R+g R)^{l}=$ (0). Diagrammatically, in the lattice, we have:

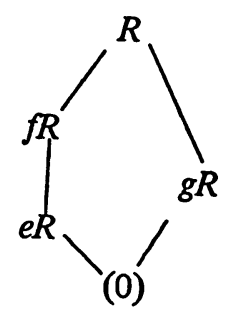

Consider the symmetric element $e+g$. If $(e+g)^{r}=t R$ for a projection $t$, 
then $e t=-g t \in e R \cap g R=(0)$, so $e t=g t=0$. Applying *, $t e=t g=0$, so $t$ belongs to $(e R+g R)^{l}=(0)$. Thus $e+g$ has zero right annihilator, so $(e+g) R$ is essential. But $f-e$ is a projection orthogonal to $e$, and we have

$$
(f-e) R \cap(e+g) R \subset(f-e) R \cap(e R \oplus g R)=(0) .
$$

Thus $f=e$, so the lattice is modular.

We can now state the main theorem of this section.

THEOREM 2.3. Let $R$ be a strongly modular Baer * ring. Then:

(1) The maximal ring of right quotients of $R, Q($ or $Q(R))$, equals the maximal ring of left quotients of $R$.

(2) The involution extends (uniquely) from $R$ to $Q$, making $Q$ into a complete *-regular ring.

(3) $R$ contains all the projections of $Q$.

(4) The inclusion $R \subset Q$ is an intimate extension of rings.

(5) $Q$ coordinatizes the projection lattice of $R$.

Further, if $R$ has no central abelian projections, then $R \subset Q$ is an epimorphism of rings, and if $R$ is additionally semihereditary, then $R \subset Q$ is a flat epimorphism of rings.

In [19], Pyle used stronger assumptions ( $L P \sim R P$, finiteness, and sufficiently many projections jointly imply strong modularity) to prove a result contained in 2.3. (1) to (5) apply even in the abelian case.

We shall prove the theorem is steps.

Recall from [14], the definitions of nonsingular ring, module, etc. It is clear that a $\mathrm{Baer}{ }^{*}$ ring is a nonsingular ring on either side.

LEMMA 2.4. Let $A, B, C$ be submodules of a nonsingular module. If $A$ is an essential submodule of $B$, then $A+C$ is an essential submodule of $B+C$.

Proof. Choose $b+c$ in $B+C$. Define the right ideal $E$ by

$$
E=b^{-1} A:=\{r \in R \mid b r \in A\} .
$$

Then $E$ is essential. As the singular submodule is zero, if $b+c$ is not zero, then $(b+c) E$ is not zero, so $(b+c) R \cap(A+C) \neq(0)$.

LEMMa 2.5. Let $R$ be a strongly modular Baer * ring. For any finite subset $\left\{t_{i}\right\}_{i=1}^{n}$ of $R, \sum t_{i} R$ is an essential submodule of $\left(\sum t_{i} R\right)^{l r}$; the left-right symmetry of this also holds.

Proof. For $n=1$, this is a consequence of 2.2(a). Consider $t_{1} R+t_{2} R$. Now $t_{i} R$ is essential in $p_{i} R$, where $\operatorname{LP}\left(t_{i}\right)=p_{i}$; by the preceding lemma, $t_{1} R+t_{2} R$ is essential in $p_{1} R+t_{2} R$, which is, in turn, essential in $p_{1} R+p_{2} R$. 
So it suffices to show $p_{1} R+p_{2} R$ is essential in $\left(p_{1} R+p_{2} R\right)^{l r}$, which equals $\left(p_{1} \vee p_{2}\right) R$, by $2.1(\mathrm{a})$.

Let $q$ be the projection such that $q R=p_{1} R \cap p_{2} R$. Then $q<p_{i}$, so for $i=1,2, p_{i}-q$ and $q$ are a pair of orthogonal projections. Thus

$$
p_{i} R=\left(p_{i}-q\right) R \oplus q R
$$

Define $p=p_{1} \vee p_{2}$. Consider the element

$$
z=1-p+p_{1}-q+p_{2}-q+q=1-p+p_{1}+p_{2}-q .
$$

Then $z$ belongs to $(1-p) R \oplus\left(p_{1}-q\right) R \oplus\left(p_{2}-q\right) R \oplus q R$ (observe that $\left.p_{1} R+p_{2} R=\left(p_{1}-q\right) R \oplus\left(p_{2}-q\right) R \oplus q R\right)$, and $z$ is symmetric. Let $e$ be the right annihilating projection of $z$. From $z e=0$, we obtain

$$
(1-p) e+\left(p_{1}-q\right) e+\left(p_{2}-q\right) e+q e=0 .
$$

Because the sum of the corresponding principal right ideals is direct, we must have

$$
(1-p) e=\left(p_{1}-q\right) e=\left(p_{2}-q\right) e=q e=0 .
$$

Applying the involution, $e(1-p)=0$, and $e p_{1}=e p_{2}=0$. Thus

$$
e \in\left(p_{1} R+p_{2} R\right)^{l}=\left(p_{1} R+p_{2} R\right)^{l r l}=p^{l}=R(1-p) .
$$

Therefore, $e=e(1-p)=0$, so $z^{r}=(0)$, and therefore $z R$ is essential. Were $p_{1} R+p_{2} R$ not essential in $p R$, there would exist a nonzero $b$ in $p R$ with $b R \cap\left(p_{1} R+p_{2} R\right)=(0)$. It is not difficult to see that

$$
\left(\left(p_{1} R+p_{2} R\right) \oplus(1-p) R\right) \cap b R=(0)
$$

(since $b R \subset p R$ ). This means $z R \cap b R=(0)$, contradicting $z R$ being essential.

Hence the lemma holds for $n=2$. The induction step is clear $\left(t_{1} R+t_{2} R\right.$ $+t_{3} R$ is essential in $p R+t_{3} R$, etc.) as in the right-left symmetry. [12]:

Before proceeding, we note the following important result of Kaplansky

Any complete orthocomplemented modular lattice is a continuous geometry.

Thus if $R$ is a strongly modular Baer * ring, $L(1, R)$ is a continuous geometry. This is the only use 2.2 (b) will be put to.

Lemma 2.6. Let $R$ be a strongly modular Baer * ring; if $I$ is a right ideal of $R$, then $I$ is an essential submodule of $I^{\text {Ir. }}$; similarly, if $I$ is a left ideal, $I$ is essential in $I^{r l}$.

Proof. Identify cardinals with initial ordinals. If the lemma is not valid, there is a smallest cardinal $\Omega$ such that if $\omega$ is a smaller ordinal, every $\omega$-generated ideal satisfies the essentiality, but there is an $\Omega$-generated right 
ideal $I$ that is not essential in $I^{l r}$. By $2.5, \Omega$ must be infinite; the map $\gamma \rightarrow \gamma+1$ gives a bijection between all the ordinals less than $\Omega$ and all the nonlimit ordinals less than $\Omega$ (identifying $\Omega$ with the smallest ordinal of its cardinality). Thus we may write $I=\Sigma r_{\alpha} R$, where $\alpha$ ranges over all the nonlimit ordinals less than $\Omega$. Define a chain of ideals, $I_{0}=(0), I_{\beta}=I_{\beta-1}+$ $r_{\beta} R$ if $\beta<\Omega$ is not a limit ordinal, and if $\beta$ is a limit ordinal, $I_{\beta}=U_{\alpha<\beta} I_{\alpha}$. Then we have $I=\cup_{\beta<\Omega} I_{\beta}$, and each $I_{\beta}$ is generated by less than $\Omega$ elements. Therefore, $I_{\beta}$ is essential in $I_{\beta}^{l r}$. Let $p_{\beta}$ denote the projection generating $I_{\beta}^{l r}$. As unions of chains preserve essentiality, we have $I=\cup I_{\beta}$ is essential in $\cup p_{\beta} R$; so if $p$ is the supremum of all the $p_{\beta}$, by Proposition 2.1(a), it suffices to show $\cup p_{\beta} R$ is essential in $p R$. As $I \cap b R=(0)$ for some nonzero $b$ in $I^{\text {lr }}$ $(=p R)$, we have $\left(\cup p_{\beta} R\right) \cap b R=(0)$. As $b$ belongs to $p R, \operatorname{LP}(b)=g$ also belongs to $p R$, so $g \leqslant p$. Also, $b R$ is essential in $g R$ by 2.2(a), so that $\left(\cup p_{\beta} R\right) \cap g R=(0)$. But the upper continuity of a continuous geometry,

$$
\begin{aligned}
g=g \wedge p & =g \wedge\left(\bigvee p_{\beta}\right) & & \\
& =\bigvee\left(g \wedge p_{\beta}\right) & & \text { (since the } p_{\beta} \text { form an increasing chain) } \\
& =(0) & & \left(\text { since } g R \cap p_{\beta} R=(0) \text { for all } \beta\right) .
\end{aligned}
$$

So $g=0$ and thus $b$ is zero, and we have arrived at a contradiction.

THEOREM 2.7 [24, THEOREM 3.3]. Let $R$ be a right and left nonsingular ring. Then the maximal ring of right quotients of $R$ equals the maximal ring of left quotients of $R$ if and only if

(a) for all right ideals $I, I^{l}=(0)$ implies $I$ is essential,

(b) for all left ideals, $I, I^{r}=(0)$ implies $I$ is essential.

Proof of Theorem 2.3, Begun. As Baer * rings are easily seen to be nonsingular on both sides, 2.7 and 2.6 apply to any strongly modular Baer * ring, so the maximal ring of right quotients equals the left, establishing (1) of 2.3.

Let us denote by $Q$ (or $Q(R)$ if there is some ambiguity), the maximal ring of quotients (the 'right' may now be dropped!). We extend the involution from $R$ to $Q$ as follows: select $q$ in $Q$; then $q$ may be regarded as a right module homomorphism from an essential right ideal $E$ of $R ; E^{*}$ is then an essential left ideal, so we define $q^{*}$ on $E^{*}$ to be $\left(e^{*}\right) q^{*}=(q(e))^{*}$. ( $q^{*}$ acts on the right.) As $Q$ is also the left maximal ring of quotients, the so-defined $q^{*}$ belongs to $Q$. It is routine to verify that this is an involution extending to the original on $R$, and uniqueness is also clear, because the extended involution must satisfy $\left(e^{*}\right) q^{\#}=(q(e))^{*}$.

To show $Q$ is *-regular, it suffices to show $q q^{*}=0$ implies $q=0$ [13, Ex. 5, p. 38]. If $q$ is not zero, there exists a $t$ in $R$ such that $t q$ is not zero and 
belongs to $R$. Then $0 \neq(t q)(t q)^{*}=t q q^{*} t^{*}$, whence $q q^{*}$ is not zero. Thus $Q$ is *-regular.

Now $Q$ is right (and left) self-injective, and therefore its lattice of principal right ideals is complete. So $Q$ is a complete *-regular ring (equivalently, $Q$ is regular and Baer *). This establishes (2) of 2.3.

LEMMA 2.8. Let $R$ be a right and left nonsingular ring, and suppose $S$ is an overring such that both ${ }_{R} S$ and $S_{R}$ (i.e. as left, right modules) are essential over ${ }_{R} R, R_{R}$, respectively. Then

(a) given a subset $W$ of $S, W^{r_{s}}=(S W \cap R)^{r_{s}}$;

(b) the map of right annihilators of $R$ to those of $S, V^{r_{R}} \rightarrow^{\beta} V^{r_{s}}$ is a lattice isomorphism, with inverse $W^{r_{s}} \rightarrow^{\alpha}(S W \cap R)^{r_{R}}$.

Proof. (a) ${ }_{R} S$ is essential over $R$, so $S W \cap R$ is left essential in $S W$. If $S$ belongs to $(S W \cap R)^{r_{R}}$, but $S W s \neq(0)$, then choose $w$ in $W$ with ws nonzero. The left ideal $E=\{t \in R \mid t w \in S W \cap R\}$ is essential, but Ews =(0); as $S$ is left nonsingular, this is a contradiction. Thus

(1) $(S W \cap R)^{r_{R}} \subset W^{r_{s}}$.

Let $V$ be any subset of $R$. If $V s=(0)$ for some $s$ in $S$, then $V(s S \cap R)=$ (0); now $s S \cap R$ is right essential in $s S$, so by the right nonsingularity, $V^{r_{R}}$ is essential in $V^{r_{s}}$. Thus $(S W \cap R)^{r_{R}}$ is right essential in $(S W \cap R)^{r_{s}}$. Taking left annihilators in $S$, we see by the right nonsingularity, that

$$
(S W \cap R)^{r_{R} l_{s}}=(S W \cap R)^{r_{s} l_{s}} .
$$

Applying $I_{s} r_{s}$ to (1), we thus obtain

$$
(S W \cap R)^{r_{s}}=(S W \cap R)^{r_{s} l_{s} r_{s}} \subset W^{r_{s}} .
$$

As $S W \cap R \subset S W$, the reverse inequality holds trivially, proving (a).

(b) It follows from (a), that $\alpha$ is a lattice map, and $\beta \alpha$ is the identity. It is easy that $\beta$ is a lattice homomorphism as well, and it is trivial to verify that $\beta$ is one-to-one. Thus $\alpha$ and $\beta$ are isomorphisms.

Proof of Theorem 2.3, Completed. Pick an annihilator right ideal in $Q$, $W^{r}$. Let $p$ be the right annihilating projection (in $R$ ) of $Q W \cap R$. It follows from the proof of 2.8 , that $p R$ is essential in $W^{r_{Q}}$, and it is easy to see that $p Q$ is also essential in $W^{r}$. But $p Q$ is itself an annihilator, so taking left, then right, annihilators, we get $W^{r}=p Q$. Now if $q$ is a projection of $Q, q Q$ is an annihilator right ideal, so there exists a projection $p$ from $R$ such that $p Q=q Q$. Thus $p=q$, verifying (3) of Theorem 2.3. As every principal right ideal of $Q$ is an annihilator ideal (since $Q$ is regular), the inclusion $\pi$ in $Q$ is therefore intimate on the right, and by the involution, is intimate. So we have (4), and (5) is a trivial consequence.

Finally, suppose $R$ has no central abelian projections. Since the centre of $R$ is contained in the centre of $Q$ and all the projections of $Q$ are already in $R$, it 
follows that $Q$ has no central abelian projections, whence by [24, Theorem 3.2], $Q$ is generated by its idempotents. By Theorem 1.9, the inclusion $R \subset Q$ is an epimorphism of rings. The flatness comes from [4, p. 246, bottom line].

We can immediately see that if a Baer * ring has a maximal ring of quotients to which the involution extends, or in which the Baer * ring is intimately embedded, then it must already be strongly modular. (The converse of 2.7 may be used.)

We can now give some readily verifiable criteria for strong modularity. But we must first establish some notation.

Let $e, f$ be idempotents in any ring $R$. We say $e$ is equivalent to $f(e \sim f)$ if $e R \cong f R$ as right $R$-modules; equivalently, there exist $x, y$ with $x y=e$ and $y x=f$. If $e, f$ are projections in a Baer * ring (or *-regular ring), then $e$ is *-equivalent to $f$ if they are linked by a partial isometry $(e \sim f)$, that is, there is an element $w$ such that $w w^{*}=e, w^{*} w=f$. Any element $w$ such that $w w^{*}$ is idempotent (and therefore a projection) is called a partial isometry, and if $x x^{*}=0$ implies $x=0$ for all $x$ (as occurs in all the rings we are working with), then $w^{*} w$ is also idempotent. The notation conforms with that in [13] but differs from that in [2].

The Baer * ring $R$ satisfies $L P \sim R P$ if $\mathrm{LP}(r) \sim \mathrm{RP}(r)$ for all $r$ in $R$ (in [2], there is no ${ }^{*}$ ). If $\operatorname{LP}(r) \sim \operatorname{RP}(r)$, for all $r$, we denote this condition by $l p \sim r p$.

A ring is directly finite if $x y=1$ implies $y x=1$. It is easily seen that a strongly modular ring is directly finite.

The following comment will also be important in $\S \S 3,4$. If $R$ is a strongly modular Baer * ring, then the maximal ring of right quotients equals the maximal ring of left quotients. Now for the ring $R$, and either right or left maximal rings of quotients, $M_{n} Q(R)=Q\left(M_{n} R\right)$, so for any matrix ring, the maximal ring of right quotients will equal the left. It follows from the easy direction of Theorem 2.7, that if $A \in M_{n} R$, then $A^{r}=(0)$ implies $A M_{n} R$ is an essential right ideal. So if $M_{n} R$ is a Baer * ring with respect to any involution (i.e., it need not depend on the involution on $R$ ), then $M_{n} R$ is strongly modular. Similarly, if $e$ is a projection of $R$ then $e R e$ is a strongly modular Baer * ring.

A Baer * ring is finite if $x x^{*}=1$ implies $x^{*} x=1$. Obviously, strong modularity implies direct finiteness, which, in turn, implies finiteness.

Proposition 2.9. Let $R$ be a Baer * ring that is isomorphic to an $n \times n$ matrix ring over some other ring, for some $n>1$.

(a) If $R$ satisfies $r^{r}=(0)$ implies $r^{l}=(0)$, that is, $\operatorname{RP}(r)=1$ implies $\operatorname{LP}(r)=$ 1 (or left zero divisors are right zero divisors), then $R$ is strongly modular, and conversely. 
(b) If $R$ satisfies $\mathrm{LP} \sim \mathrm{RP}$ and is a finite Baer * ring, then $R$ is strongly modular.

(c) If $R$ satisfies $\mathrm{lp} \sim \mathrm{rp}$ and is directly finite, then $R$ is strongly modular.

Proof. By hypothesis, there exist orthogonal idempotents with $\left\{e_{i}\right\}_{i=1}^{n}$, $\sum e_{i}=1$, and $e_{i} \sim e_{j}$. Let $p$ denote the left projection of $l_{1}$, so $p R=e_{1} R$. Then if $S=e_{1} R e_{1} \simeq p R p, S$ possesses an involution making it into a Baer * ring, and we may regard $R$ as $M_{n} S$ (however, the involutions on $R$ and $S$ may have nothing to do with each other). To begin with, we show $S$ is strongly modular. Find $a$ in $S$ such that $a^{r}=(0)$. Suppose there exists $b$ in $S$ such that $a S \cap b S=(0)$. As $S$ is a Baer ring, there exists an idempotent $e$ in $S$ such that $e S=b^{r}$. Consider the matrix

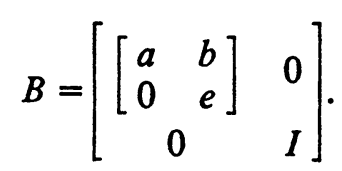

( $I$ is the identity matrix of size $n-2$.) Regarding $B$ as an element of $R$, and using $a S \cap b S=(0)$ and $a^{r}=(0)$, we obtain $B^{r}=(0)$. However, it is easily seen that

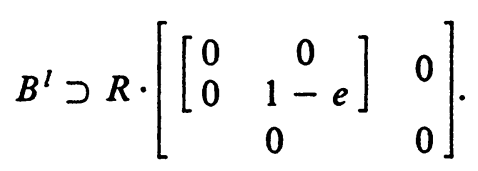

The hypothesis tells us $1-e=0$, so $b=0$, and thus $S$ is strongly modular. By the comment preceding this proposition, $R=M_{n} S$ is strongly modular. The converse is trivial, and (b) and (c) are immediate consequences of (a).

Of course the $n \times n$ matrix condition is essential. Let $R$ be any non-Ore domain with an involution. Then $R$ is vacuously a Baer ${ }^{*}$ ring (even directly finite), with $L P \dot{\sim}_{R P}$, but $R$ is not strongly modular. Proposition 2.9 will figure heavily in $\$ \S 3,4$.

A Baer * ring has sufficiently many projections [19] if every nonzero right ideal contains a nonzero projection.

Proposition 2.10. Let $R$ be a Baer * ring having sufficiently many projections. Then $R$ is stongly modular if and only if $a^{r}=(0)$ implies $a^{l}=(0)$, i.e., $\operatorname{RP}(a)=1$ implies $\operatorname{LP}(a)=1$. In particular, $a$ finite Baer * ring having sufficiently many projections and satisfying $\mathrm{LP} \sim \mathrm{RP}$ is strongly modular.

Proof. If $a^{r}=(0)$ implies $a R$ is essential, then $a^{l}=(0)$, as the ring is nonsingular.

Conversely, suppose $a^{r}=(0)$ but $a R \cap c R=(0)$ for some nonzero $c$ in $R$. There exists a nonzero projection $p$ such that $p$ is in $c R$, so that $a R \cap p R=$ 
(0). If $(a-p a) t=(0)$, then $a t=p a t \in a R \cap p R=(0)$, so $a t=0$, and thus $t=0$. Hence $(a-p a)^{r}=(0)$; however, $p(a-p a)=(0)$, contradicting the hypothesis.

It follows that a finite $A W^{*}$ algebra (real or complex) is strongly modular.

3. Matrix rings. In this section, we make significant progress on the problem of matrix rings over Baer * rings (when are they Baer ${ }^{*}$ ?). A considerable improvement on the previously known result is obtained [2, Theorem 1, p. 262]. The maximal ring of quotients is employed throughout.

A Baer * ring satisfies GC $[2, \S 14]$ if for all pairs $e, f$ of projections, there eixsts a central projection $h$ such that $h e$ is *-isomorphic to a subprojection of $h f$ and $(1-h) f$ is *-isomorphic to a subprojection of $(1-h) e$.

A Baer * ring satisfies SR [2, p. 66] if for all $x$ in $R$, there exists an $r$ in $R$ such that $r=r^{*}, r^{2}=x^{*} x$, and $r$ commutes with everything commuting with $x^{*} x$.

Suppose $R$ is a finite Baer * ring with the following properties:

(a) either LP $\sim \mathrm{RP}$ or SR holds;

(b) $R$ is isomorphic to an $n \times n$ matrix ring over another ring for some $n>3$.

These conditions are weaker than they appear. Condition (b) is automatically satisfied (in the presence of (a)) if $R$ is of type II, so the $n>3$ condition applies only in the type I case. If $R$ possesses sufficiently many projections, then the $n>3$ condition can be reduced to no central abelian projections, in what follows.

We shall show that if $R$ satisfies these conditions, then for all $m, M_{m} R$ is a Baer * ring with respect to *-transpose, and it follows immediately that $M_{m} R$ is strongly modular, and therefore finite. In this section, only $L P \sim R P$ will be dealt with; in $\S 5$, it will be shown that $S R$ implies $L P \sim R P$ (in the presence of finiteness and (b)).

The first step in proving the advertised results is to show that in a strongly modular Baer * ring, $\mathrm{LP} \sim \mathrm{RP}$ extends to the maximal ring of quotients.

LEMMA 3.1. In $a$ *-regular ring, the following are equivalent:

(a) $\mathrm{LP} \sim \mathrm{RP}$,

(b) $e \sim$ fimplies $e \dot{\sim} f$.

Proof. If $e \sim f$, there exist $x \in e R f, y \in f R e$, such that $x y=e$, and $y x=f$. Trivially, $\operatorname{LP}(x)=e, \operatorname{RP}(x)=f$.

Conversely, choose $r$ in $R$ and set $e=\mathrm{LP}(r), f=\mathrm{RP}(r)$. Now, $r R=e R$ (since we are in a regular ring), but as $r^{r}=(1-f) R$, and $r R \cong R / r^{r}$ as right $R$-modules, $r R \cong f R$, so $e R \cong f R$; but this is the same as $e \sim f$. 
Proposition 3.2. Let $R$ be a complete *-regular (= regular Baer *) ring with GC. Then for projections $e, f$, in $R, e \sim f$ implies $e \sim f$. In particular, $\mathrm{LP} \sim \mathrm{RP}$ holds.

Proof. Suppose $e \sim f$. By [2, Proposition 1, \$14], we may orthogonally decompose $e=e_{1}+e_{2}, f=f_{1}+f_{2}$ where $e_{1} \sim f_{1}$ and $f_{2} R e_{2}=(0)$. Now, $e_{1} \sim f_{1}$, and as $R$ is a continuous ring [9], by [23, Theorem 5.6], $e_{2} \sim f_{2}$. But this contradicts $f_{2} R e_{2}=(0)$, unless $e_{2}=f_{2}=0$, and so $e \dot{\sim}^{*} f$.

PRoposition 3.3. If $R$ is a strongly modular Baer * ring satisfying $\mathrm{LP} \sim \mathrm{RP}$, then its maximal ring of quotients also satisfies $\mathrm{LP} \sim \mathrm{RP}$.

Proof. From [2, Corollary 2, p. 80], $R$ satisfies GC. Now the centre of $R$ is contained in the centre of $Q$ (trivial), and $Q$ has no new projections by Theorem 2.3(3), so $Q$ also satisfies GC. By 3.2, $Q$ also has LP $\sim$ RP.

Let $R$ be a *-ring satisfying $\sum r_{i} r_{i}^{*}=0$ implies all the $r_{i}$ are zero. An element $r$ of $R$ is bounded [25], [2, p. 243] if there exist $s_{i}$ in $R$ such that $r r^{*}+\sum s_{i} s_{i}^{*}$ is a rational number. The collection of all bounded elements is a subring containing all the projections, partial isometries of $R$; we will denote this subring by $R_{b}\left(R_{0}\right.$ in [2]).

LEMMA 3.4. Let $R$ be a strongly modular Baer * ring such that

$$
\sum r_{i} r_{i}^{*}=0 \text { implies all the } r_{i} \text { are zero. }
$$

Then:

(a) $Q$, the maximal ring of quotients of $R$, also satisfies (A).

(b) $M_{n} Q$ is a Baer * ring for all $n$.

(c) $\left(M_{n} Q\right)_{b} \subset M_{n} Q_{b}$.

(d) If $Q_{b} \subset R$, then $M_{n} R$ is a Baer * ring for all $n$ (with respect to *-transpose), and $M_{n} R$ contains all the partial isometries of $M_{n} Q$.

Proof. (a) If $\Sigma q_{i} q_{i}^{*}=0$ but $q_{1}$ is not zero, we may find $r_{1}$ in $R$ such that $r_{1} q$ is not zero and lies in $R$. Then $\Sigma\left(r_{1} q_{i}\right)\left(r_{1} q_{i}\right)^{*}=0$. If $r_{1} q_{2}$ does not belong to $R$, we may find $r_{2}$ in $R$ such that $r_{2} r_{1} q_{2}$ is not zero and lies in $R$. Continuing in the obvious manner, we eventually reach a contradiction.

(b) $Q$ is self-injective regular, so $M_{n} Q$ is self-injective regular, and therefore is a Baer ring. Now $M_{n} Q$ is *-regular by Theorem 2.3 and [13, Ex.5, p. 38]. A Baer ring that is *-regular is trivially a Baer * ring!

(c) If $A$ belongs to $M_{n} Q$ and is bounded, there exist $B_{i}$ in $M_{n} Q$ such that

$$
A A^{\#}+\sum B_{i} B_{i}^{\#}=\beta I,
$$

(\# denotes *-transpose), $\beta$ a rational; then solving these equations in terms 
of the entries of $A$, we see that all of the entries are bounded, and so the inclusion holds.

(d) All partial isometries (and therefore all projections) of $M_{n} Q$ are bounded (since $\left.w w^{*}+\left(1-w w^{*}\right)\left(1-w w^{*}\right)^{*}=1\right)$, so $M_{n} R$ contains all the partial isometries of $M_{n} Q$, by (c). As $M_{n} Q$ is Baer ${ }^{*}$, it easily follows that $M_{n} R$ is a Baer * ring (it is sufficient that it contain all the projections of $\left.M_{n} Q\right)$.

Using this lemma, it can be shown that if $X$ is an extremely disconnected compact space, and $C(X)$ is the ring of all real, complex or quaternion-valued continuous functions, then $M_{n} C(X)$ is Baer *. (Of course, this is well known, but the above lemma provides an especially easy proof.)

Implicit in much of what follows is the result that if $R$ is strongly modular and $R=M_{n} S$ for some $n$, then $S$ is strongly modular, and $M_{n} Q(S)$ can be naturally identified with $Q(R)$.

From now until the end of $\S 4, R=M_{n} S$ will indicate $R$ is a matrix ring over $S$, with involution *-transpose, where ${ }^{*}$ is an involution on $S$.

$Q$ will denote the rational numbers.

LEMMA 3.5. Let $R$ be a strongly modular Baer * ring.

(a) If $R=M_{2} S$, then $S$ contains all the partial isometries of $Q(S)$.

(b) If $R=M_{4} T$, and $T$ satisfies the following properties:

(i) $T$ is an algebra over $\mathbf{Q}$,

(ii) for all positive $\alpha$ in $\mathbf{Q}$, there exists $d$ in $T$ such that $d d^{*}=d^{*} d=\alpha$; then, if $a, b$ belong to $Q(T)$ and satisfy $a a^{*}+b b^{*}=\alpha \in Q$, then $a, b$ belong to $T$.

Proof. (a) We know $R$ contains all the projections of $Q(R)=M_{2} Q(S)$. Let $w$ be a partial isometry of $Q(S)$. Then 2 is invertible in $Q(R)$ [2, Lemma 2 , p. 56], hence in $Q(S)$, so

$$
p=\frac{1}{2}\left[\begin{array}{cc}
w w^{*} & w \\
w^{*} & w^{*} w
\end{array}\right]
$$

is a projection in $Q(R)$. As $p$ belongs to $R, w$ belongs to $S$.

(b) Set $S \stackrel{*}{=} M_{2} T$; then $S$ is itself a Baer * ring. We may find $d$ in $T$ such that $d d^{*}=d^{*} d=\alpha$ (as the involution is proper, it is easy to see that $\alpha>0$ if $a$ is not zero). Since $\alpha$ is a unit in $T$ and $T$ is directly finite (being strongly modular), $d^{-1}$ belongs to $T$. Consider the element, $w$, of $Q(S)$ defined by

$$
w=\left[\begin{array}{cc}
a d^{-1} & b d^{-1} \\
0 & 0
\end{array}\right]
$$

Then $w w^{* t}$ is a projection, so $w$ is a partial isometry, and thus by (a), w belongs to $S=M_{2} T$. Thus $a=\left(a d^{-1}\right) d$ belongs to $T$. 
LEMMA 3.6 [18]. Let $R$ be $a^{*}$-regular ring satisfying LP $\sim$ RP.

(1) If $g$ is a projection such that $g$ is *-isomorphic to a subprojection of $1-g$, then the ring $g R g$ satisfies:

For all $c, d$ in $g R g$, there exists $b$ in $g R g$ such that $b b^{*}=c c^{*}$ $+d d^{*}$.

(2) If $R$ has no central abelian projections, then $R$ satisfies condition (A) of Lemma 3.4, and thus $M_{n} R$ is *-regular for all $n$.

Proof. (1) [18, Lemma 1] and 3.1.

(2) [18, Corollary, p. 217] and 3.1.

To avoid a rather awkward additional hypothesis (cf. Lemma 3.5(b)), we prove the following odd lemma.

LEMMA 3.7. Let $R$ be a strongly modular Baer ${ }^{*}$ ring satisfying $\mathrm{LP} \sim \mathrm{RP}$, and suppose that $R=M_{2} S$. Then

(a) $S$ (and therefore $R$ ) is an algebra over the rational numbers;

(b) for every positive rational number $\alpha$, there exists $c$ in $S$ such that $c c^{*}=c^{*} c=\alpha$.

Proof. We first observe that by $3.3, Q(R)$ satisfies $\mathrm{LP} \sim \mathrm{RP}$; since $Q(R)=M_{2} Q(S), Q(R)$ contains no central abelian projections, and thus by 3.6(1), $r \neq 0$ implies $n r r^{*} \neq 0$ for any integer $n>0$. Thus $n$ is not a zero divisor in $Q(R)$, so by the regularity of $Q(R), n$ must be invertible in $Q(R)$. Therefore $Q(R)$ is a Q-algebra, and by Morita equivalence, $Q(S)$ is also a Q-algebra.

Now, given a positive integer $n$, by $3.6(1)$, there exists $c$ in $Q(S)$ such that $c c^{*}=n$. Thus $(c / n) c^{*}=1$. By direct finiteness, $c$ and $c^{*}$ commute. Set $k=c / n$. Then $k k^{*}=k^{*} k=1 / n$. Consider, the element $p$ of $M_{2} Q(S)=$ $Q(R)$ defined by

$$
p=\frac{1}{1+(1 / n)}\left[\begin{array}{cc}
1 & k^{*} \\
k & 1 / n
\end{array}\right]
$$

Then $p=p^{* t}$, and $p^{2}=p$ follows from $k$ commuting with $k^{*}$. Thus $p$ is a projection of $Q(R)$, so $p$ belongs to $R=M_{2} S$ by 2.3. Hence, $n /(n+1)$ and $1 /(n+1)$ belong to $S$ for all integers $n>0$, so $S$ is indeed a $\mathbf{Q}$-algebra. Finally, $k /(1+(1 / n))$ belongs to $S$; we know $S$ is a Q-algebra, so $k$ belongs to $S$. Set $c=n k$; we have $n=c c^{*}$, and it easily follows that every positive rational has a 'square root' in the sense of (b).

THEOREM 3.8. Let $R$ be a finite Baer * ring with the following properties:

(a) $\mathrm{LP} \sim \mathrm{RP}$ 
(b) $R$ possesses $m$ equivalent orthogonal projections adding to 1 , for some $m>3$.

Then: (1) $R$ is strongly modular,

(2) $M_{n} R$ is Baer * with respect to *-transpose, for all $n$, and is strongly modular,

(3) For all $n$ (including $n=1$ ), all partial isometries of $M_{n} Q$ belong to $M_{n} R$, where $Q$ is the maximal ring of quotients of $R$.

Proof. (1) This is Proposition 2.9: (b) is equivalent to $R=M_{m} T$.

(2) In the presence of (a), $e \sim f$ implies $e \sim f$ (see the proof of 3.1). Thus from the second hypothesis, there exist a set of $m$ projections $\left\{e_{i}\right\}$ such that $e_{i} \sim e_{j}$ and $e_{i} R \cap e_{j} R=(0)$ if $i \neq j$. Denote by $p^{n}, \sup \left\{e_{i} \mid i<n\right\}$. It is easy to see that $\left\{p^{n}-p^{n-1}\right\}$ form an orthogonal set of projections, each of which is *-equivalent to $e_{1}$. Thus $R=M_{m} T$. By reducing to a suitable projection, we may assume $m=4$, and we have $Q(R) \stackrel{*}{=} M_{4} Q(T)$. Trivially $T$ satisfies (a) (since $T$ is *-isomorphic to $f R f, f$ a projection from $R$ ), so $Q(T)$ does by 3.3, as does $Q(R)$. Applying 3.6(1), for any bounded element $a$ of $Q(T)$, there exist $b$ in $Q(T)$ and $\alpha$ in $\mathbf{Q}$ such that $a a^{*}+b b^{*}=\alpha$. By 3.7 and 3.5(b), $a$ belongs to $t$; that is, $Q(T)_{b}$ is contained in $T$. By 3.4(d), all matrix rings over $T$ satisfy (2) and (3), and therefore so do all matrix rings $R$.

COROllary 3.9. Let $R$ be a finite Baer * ring of type II that satisfies $\mathrm{LP} \sim \mathrm{RP}$. Then all matrix rings over $R$ are Baer ${ }^{*}$ with respect to ${ }^{*}$-transpose.

Proof. By [2, Corollary, p. 121], $R$ satisfies 3.8(b) for all integers $m$.

COROLlaRY 3.10. Let $R$ be a strongly modular Baer * ring that is an $m \times m$ *-matrix ring for some $m>3$, and satisfies GC. Then $R$ satisfies $\mathrm{LP} \sim \mathrm{RP}$, and so Theorem 3.8 applies.

Proof. $Q$ satisfies GC as in the proof of 3.3 , so by $3.2, Q$ satisfies $L P \sim R P$. Now go through the proof of 3.8(3) with $n=1$, to show LP RP holds in $R$ (by showing all the partial isometries of $Q$ lie in $R$, and that $\operatorname{LP}(r)$ is the same whether taken in $R$ or $Q$ ).

Finiteness, GC, and condition (b) of 3.8 do not jointly imply strong modularity (see Example 2 of \$6).

As promised, with additional conditions on $R$, the $m>3$ in 3.8(b) can be weakened.

COROLlaRY 3.11. Let $R$ be a finite Baer * ring satisfying

(a) $\mathrm{LP} \sim \mathrm{RP}$,

(b) "sufficiently many projections", 
(c) $R$ has no central abelian projections.

Then $M_{n} R$ is a Baer * ring for all $n$.

Proof (Outline). By $2.10, R$ is strongly modular. Now, condition (c) extends to $Q$, as the centre of $R$ is contained in the centre of $Q$, and $Q$ contains no new projections; and as $Q$ is a complete *-regular ring, it means $Q=\pi Q_{i}$, where each $Q_{i}$ is a ${ }^{*}$-matrix ring of size two or larger (see e.g. [18]). So we may assume $Q$ is a $2 \times 2$ matrix ring.

Let $E$ be an essential right ideal of $R$; we may find $\left\{p_{i}\right\}$, a maximal set of orthogonal projections inside $E$. Set $p=\sup p_{i}$. It is implicit in the proof of 2.6, that $\oplus p_{i} R$ is essential in $p R$. If $p \neq 1$, then $(1-p) R \cap E$ contains a nonzero projection orthogonal to all of the $\dot{p}_{i}$ (using (b)); this is a contradiction, so $p=1$, that is, $\bigoplus p_{i} R$ is essential. Now we mimic the proof of [2, Theorem 1 and Corollary, p. 223] to show all the unitaries and all partial isometries of $Q$ are already in $R$.

Let $u$ be a unitary of $Q$; there exists an essential right ideal $E$ such that $u E \subset R$. We may find the $\left\{p_{i}\right\}$ as in the preceding paragraph, so that $\bigoplus p_{i} R$ is contained in $E$ and is essential. Define $w_{i}=u p_{i}$. Then $w_{i}^{*} w_{i}=p_{i}$ (as $u u^{*}=u^{*} u=1$ ); on the other hand $\left\{q_{i}=w_{i} w_{i}^{*}\right\}$ is an orthogonal set of projections. Now $Q$, being a matrix ring, contains no central abelian projections, so that by [2, Theorem 1(ii), p. 129], partial isometries are addable, (in $R$ ) that is, there exists $v$ in $R$ such that $v p_{i}=w_{i}=u p_{i}$. Thus $(v-u)$ annihilates $\bigoplus p_{i} R$; since this direct sum is essential, $v=u$, so $u$ belongs to $R$.

Now let $w$ be a partial isometry in $Q$. If $p=w w^{*}, q=w^{*} w$, then $p$ and $q$ are projections, so belong to $R$, and also $p \sim q$ in $Q$ by definition. As GC holds in $Q, 1-p \sim 1-q$ by $\left[2, \S 17\right.$, Proposition 4]; if $z z^{*}=1-p$ and $z^{*} z=1-q$, then $w+z$ is a unitary, so belongs to $R$. But $w=(w+z) q$, hence belongs to $R$. In particular, we obtain $R=M_{2} T$ for some $T$.

Finally, apply the proof of Lemma 3.5(b) combined with 3.7, to show all the bounded elements of $Q(T)$ lie in $T$, so 3.4(d) applies.

COROLlaRY 3.12. Let $R$ be a real $A W^{*}$ algebra without central abelian projections. Then matrix rings over $R$ are real $A W^{*}$-algebras.

Proof. $R$ splits into a product $R_{1} \times R_{2}$, where $R_{2}$ is 'purely infinite', and $R_{1}$ is finite. Thus ([2, p. 104] for example), $M_{n} R_{2} \cong R_{2}$ already. So we may assume $R$ is finite. $M_{n} R$ is well known to be a $C^{*}$-algebra, even in the real case, so the problem reduces to showing $M_{n} R$ is Baer *. But it is well known (and easy to verify) that even real $A W^{*}$ algebras have sufficiently many projections, and satisfy $\mathrm{LP} \sim \mathrm{RP}$. Thus Corollary 3.11 applies.

Of course, the 'real at infinity' $A W^{*}$ algebra [2, Ex. 1, 4, p. 131] shows 
central abelian projections can be obstructions to matrix ring theorems.

COROllary 3.13. Let $R$ be a finite Baer * ring satisfying

(a) SR,

(b) $R$ has $m$ orthogonal equivalent projections adding to 1 , for some $m>3$.

If $R$ is strongly modular, then all matrix rings over $R$ are Baer * and $R$ satisfies LP $\sim$ RP.

Proof. By [16, Theorem 2.1], $R$ has GC, so Corollary 3.10 applies.

It will be shown in $\S 5$ that the strong modularity assumption in 3.13 is redundant.

Let us see how these results compare with the previously known result. If $R$ is a finite Baer * ring satisfying $\left(1^{\circ}\right)$ through $\left(8^{\circ}\right)$ of $[2$, pp. 249,256$]$, then it was shown that $M_{n} R$ was Baer ${ }^{*}$ for all $n$ [2, Theorem 1, p. 262]. Our condition (a) of the introductory paragraphs of this section is considerably weaker than $\left(1^{\circ}\right)$. Our (b) is somewhat stronger than $\left(2^{\circ}\right)$, though not by very much (and in the type II case, both are vacuous). ( $3^{\circ}$ ) is actually a consequence of (a) and (b) [25, Lemma 6]! Conditions $\left(4^{\circ}, 5^{\circ}, 6^{\circ}\right)$ appear to have no connection with the problem. $\left(7^{\circ}, 8^{\circ}\right)$ are conditions on the matrix rings rather than the ring itself.

It is quite likely, in view of the proofs, that LP $\sim \mathrm{RP}$ could be weakened to its consequences:

(a1) strong modularity,

(a2) for all $a, b$ in $R$ there exists $c$ with $c c^{*}=a a^{*}+b b^{*}$.

An accidental consequence of $2.3,3.8$ and a result to be proved in $\S 5$, is that all the rings dealt with here turn out to be semihereditary, and therefore the inclusion $R \rightarrow Q$ is a flat epimorphism of rings.

4. Matrix rings and $L P \sim R P$. In this section, we show that the property $\mathrm{LP} \sim \mathrm{RP}$ extends to matrix rings of complete *-regular rings without central abelian projections. This section too, answers some questions in [2]. It is based on a generalization of a result of Burke on complete *-regular factors of type II.

A regular ring is unit regular (e.g. [8]) if for all $r$ in $R$, there exists a unit $u$ such that $r u r=r$. The following properties of unit regular rings are relevant to our discussion:

(i) If $e, f$ are idempotents and $e \sim f$, then $1-e \sim 1-f$ (e.g. [8, Theorem 2]). In particular, direct finiteness holds.

(ii) Matrix rings and rings of the form $e R e$ ( $e$ an idempotent) over unit regular rings are unit regular (e.g. [8, Corollary 4]).

(iii) A right and left self-injective regular ring is unit regular (e.g. [8, Corollary 7]). 
From (iii), a strongly modular Baer * ring has its maximal ring of quotients unit regular.

COROLlaRY 4.1. Let $R$ be $a^{*}$-regular unit regular ring satisfying LP $\sim$ RP. If $e, f$ are projections and $e \sim f$, then there exists a unitary $u$ that ueu* $=f$; in particular, $1-e \sim 1-f$.

Proof. From 3.1, $e \dot{\sim} f$, and by (i) above, $1-e \sim 1-f$; applying 3.1 again, $1-e \dot{\sim} 1-f$, if $w, v$ are partial isometries implementing these ${ }^{*}$-isomorphisms, then it is routine to see that $u=w+v$ is the desired unitary.

I know of no *-regular rings that are not unit regular. A complete *-regular ring is a continuous ring, and is therefore unit regular.

We show that if $R=M_{2} S$ (or any larger size matrix ring) and $R$ satisfies $\mathrm{LP} \sim \mathrm{RP}$, then all matrix rings over $R$ satisfy LP $\sim \mathrm{RP}$ (we already know that matrix rings over $R$ are *-regular, by 3.6(2)). In [3], Burke showed this holds for all complete *-regular factors of type II, which, of course, turns out to be a very special case of the result here. Our techniques are essentially the same as Burke's, but we have some simplifications that avoid reliance on either completeness, or the existence of a rank function. In [3], LP $\sim \mathrm{RP}$ is referred to as 'Property (PU)'.

LEMMA 4.2. Let $R$ be $a^{*}$-regular ring satisfying LP $\sim$ RP. If $e$ is a projection, the *-regular ring eRe also satisfies $\mathrm{LP} \sim \mathrm{RP}$.

Proof. The obvious involution on $e R e$ makes $e R e^{*}$-regular ( $t t^{*} \neq 0$, etc.). Let $p \sim q$ in $e R e$, for projections $p, q$. The equational nature of equivalence ( $x y=p, y x=q$, some $x, y$ ) shows $p \sim q$ in $R$, and therefore $p \sim q$ in $R$. Thus there exists $w$ in $R$ with $w w^{*}=p, w^{*} w=q$, and it is easy to see that $w=p w q$. But $p, q$ belong to $e R e$, so $w=e p w q e(e p=p$, etc.) belongs to $e R e$, and so the *-equivalence is implemented in $e R e$, which proves the result, by 3.1.

If $e, f$ are idempotents in any regular ring, then a monomorphism $e R \lesssim f R$ splits, i.e. there exists an idempotent $g$ in $f R$ such that $e \sim g$. We shall denote this relation between $e$ and $f$ by $e \lesssim f$. If $R$ is *-regular, and $e, f$ are projections, then we may assume $g$ is a projection (otherwise take $\operatorname{LP}(g)=h$; then $h R=g R$, so $g \sim h$ ). If there is a subprojection $g$ of $f$ such that $e \sim g$, then we shall regrettably have to denote this relationship by $e \lesssim f$.

Lemma 4.3 (Essentially [3, Lemma 3.1]). Let $S$ be a ${ }^{*}$-regular and unit regular ring satisfying $\mathrm{LP} \sim \mathrm{RP}$, and suppose there exists a projection e in $S$ such that $e \dot{\sim}-e\left(\right.$ so $\left.S=M_{2}(e S e)\right)$. Set $R \stackrel{*}{=} M_{2} S$, and let $P$ be a projection 
of $R$ such that $P \lesssim\left[\begin{array}{ll}e & 0 \\ 0 & 0\end{array}\right]$. Then $P \lesssim\left[\begin{array}{ll}e & 0 \\ 0 & 0\end{array}\right]$.

Proof. As $S$ is a $2 \times 2$ matrix ring, it has no central abelian projections, so by $3.6(2), R$ is *-regular.

Write $P=\left[\begin{array}{ll}a & b \\ c & d\end{array}\right]$ (of course $b=c^{*}$, but we do not use this), and set

$$
E=\left[\begin{array}{ll}
e & 0 \\
0 & 0
\end{array}\right], \quad E^{\prime}=\left[\begin{array}{ll}
0 & 0 \\
0 & e
\end{array}\right]
$$

Let $f_{1}$ be the projection generating $a S+b S$ (i.e., $f_{1} S=a S+b S$ ), and set

$$
F_{1}=\left[\begin{array}{cc}
f_{1} & 0 \\
0 & 0
\end{array}\right]
$$

Now it is clear that

$$
F_{1} P=\left[\begin{array}{ll}
a & b \\
0 & 0
\end{array}\right], \text { and }\left[\begin{array}{ll}
a & b \\
0 & 0
\end{array}\right] \cdot R=F_{1} R
$$

(that is, as right $R$-ideals).

Similarly, let $f_{2}$ be the projection in $S$ such that $f_{2} S=c S+d S$. and define

$$
F_{2}=\left[\begin{array}{ll}
0 & 0 \\
0 & f_{2}
\end{array}\right] \text {. }
$$

It also follows that $F_{2} P=\left[\begin{array}{ll}0 & 0 \\ c & d\end{array}\right]$, and $F_{2} R=F_{2} P \cdot R$. Now the map $P R \rightarrow F_{2} R$ of right $R$-modules given by left multiplication by $F_{2}$ is onto, and as all maps between finitely generated projective modules over a regular ring split, there is an idempotent, and therefore a projection, $Q<P$ such that $Q \sim F_{2}$ (if $Q$ is merely an idempotent, it may be replaced by its left projection, which generates the same right ideal, and therefore is equivalent to $Q$ ). Thus $F_{2} \sim Q \lesssim E \sim E^{\prime}$, so $F_{2} \lesssim E^{\prime}$. Therefore, there exist $X \in E^{\prime} R F_{2}, Y \in$ $F_{2} R E^{\prime}$ such that $Y X=F_{2}$. But the form of all the matrices in $E^{\prime} R F_{2}$ and $F_{2} R E^{\prime}$ (matrices of the form [ $\left[\begin{array}{l}0 \\ 0\end{array}\right]$ ) shows in fact $f_{2} S \lesssim e S$; since $S$ is regular, $f_{2} \lesssim e$. By unit regularity $e \sim 1-e \lesssim 1-f_{2}$. The same argument shows $F_{1} \lesssim P$, so $F_{1} \lesssim E$, and thus $f_{1} \lesssim 1-f_{2}$. By 4.1 , there is a unitary $u$ such that $u f_{1} u^{*}=h$ is a subprojection of $1-f_{2}$; in particular, $h$ and $f_{2}$ are orthogonal. Define the element $U$ of $R$ by

$$
U=\left[\begin{array}{cc}
\left(1-f_{2}\right) u & f_{2} \\
f_{2} u & 1-f_{2}
\end{array}\right]
$$

Then $U U^{* t}=I$, so by direct finiteness (from $S$ and therefore $R$ being unit regular), $U$ is unitary. Now we have

$$
f_{2} u a+\left(1-f_{2}\right) c=f_{2} u f_{1} u^{*} u a+\left(1-f_{2}\right) f_{2} c=0
$$

(as $\left.f_{2} u f_{1} u^{*}=0\right)$ and also $f_{2} u b+\left(1-f_{2}\right) d=0$ (for the same reasons). Thus the bottom row of the matrix UP is just $(00)$. Therefore the bottom row of 
$U P U^{* t}=H$ is also $(00)$. However, $H$ is a projection and therefore ${ }^{*}$-transpose symmetric, so $H$ is of the form $H=\left[\begin{array}{l}g \\ 0\end{array}\right]$, for some projection $g$ in $S$. Thus we have $P \sim H$, and as $P \lesssim E$, we get $H \lesssim E$; and as in the above paragraphs, this gives us $g \lesssim e$; by $\mathrm{LP} \sim \mathrm{RP}, g \lesssim e$. Now it is trivial to verify

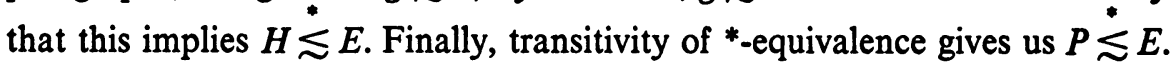

LEMma 4.4. Let $R \stackrel{*}{=} M_{2} S$, where $S$ is *-regular and unit regular. Suppose there exists a projection $e$ in $S$ such that $e \sim_{1}-e$. Then if $P$ is a projection of $R, P$ may be written as the sum of 4 orthogonal projections, $P=P_{1}+P_{2}+P_{3}$ $+P_{4}$ such that for each $i=1,2,3,4, P_{i} \lesssim\left[\begin{array}{l}e \\ 0\end{array}\right]$.

Proof. We have $S \stackrel{\bullet}{=} M_{2} T$, where $T=e S e$ (in a natural manner). Now $P \in M_{4} T$, so $P$ may be regarded as a map of $T$-modules $4 T \rightarrow 4 T$, where $4 T$ denotes the free $T$-module on 4 generators. Now $P(4 T)$ is a finitely generated submodule of a projective module over the regular ring $T$, so is a direct summand, and projective. By [14, Proposition 8, p. 85], there exist submodules (necessarily projective and finitely generated) such that $P(4 T)=\bigoplus^{4} N_{i}$, where each $N_{i}$ is isomorphic to a principal right ideal of $T$, as $T$-modules. We may therefore find orthogonal idempotents $E_{i}$ (corresponding to the $N_{i}$ ) such that $P=E_{1}+E_{2}+E_{3}+E_{4}$, and $E_{i} \lesssim\left[\begin{array}{l}e \\ e \\ 0\end{array}\right]$, the latter of which corresponds to a copy of $T$ inside $4 T$. Now set $P^{i}$ to be the left projection of $E_{1}+\cdots+$ $E_{i}$, and define $P_{i}=P^{i}-P^{i-1}\left(P^{0}=0\right)$. Now $P_{1} R=E_{1} R$, and $P_{2} R \oplus P_{1} R$ $=E_{1} R \oplus E_{2} R$; by the unit regularity of $P^{2} R P^{2}, P_{2} \sim E_{2}$; similarly, $P_{i} \sim E_{i}$. As $P^{4}=P$, and the $P_{i}$ are obviously orthogonal, we have the result.

THEOREM 4.5. Suppose $S$ is $a^{*}$-regular and unit regular ring such that $S=M_{m} T$ for some $m>1$, some ${ }^{*}$-ring $T$. If $S$ satisfies $\mathrm{LP} \sim \mathrm{RP}$, then for all $n, M_{n} S$ is $a^{*}$-regular ring (with respect to *-transpose) satisfying $\mathrm{LP} \sim \mathrm{RP}$.

Proof. By 3.6(2), $M_{n} S$ is *-regular for all $n$.

Now we note that it suffices to prove that $M_{2} S$ has LP $\sim$ RP: For, assuming this result, inductively we have $M_{2^{n}} S$ has the property, and so $M_{n} S=e \cdot M_{2^{n}} S \cdot e$ has it by 4.2. A similar reduction allows us to assume $m=2$. Set $R=M_{2} S$. Choose a projection $P$ in $R$. By 4.4 and 4.3, there is an orthogonal decomposition $P=P_{1}+P_{2}+P_{3}+P_{4}$ into projections, where each $P_{i}$ is *-equivalent to a subprojection of $\left[\begin{array}{l}e \\ 0 \\ 0\end{array}\right]$ in $R$, where $e$ is the projection such that $e \sim 1-e$ (i.e., $T=e S e$ ). As

$$
\left[\begin{array}{ll}
0 & 0 \\
0 & e
\end{array}\right] \sim\left[\begin{array}{ll}
e & 0 \\
0 & 0
\end{array}\right] \sim\left[\begin{array}{cc}
1-e & 0 \\
0 & 0
\end{array}\right] \div\left[\begin{array}{cc}
0 & 0 \\
0 & 1-e
\end{array}\right]
$$


trivially, the $P_{i}$ are *-equivalent to an orthogonal family of diagonal projections. By the additivity of *-equivalence [13, Theorem 25, p. 33], $P$ is *-equivalent to a diagonal projection of the form

$$
\left[\begin{array}{cc}
f_{1}+f_{2} & 0 \\
0 & f_{3}+f_{4}
\end{array}\right]
$$

where $f_{1}, f_{2}$ are orthogonal projections in $S$, as are $f_{3}, f_{4}$.

$$
\left(P_{1} \sim\left[\begin{array}{ll}
f_{1} & 0 \\
0 & 0
\end{array}\right], \text { etc. }\right)
$$

Let $Q$ be any other projection in $R$, and suppose $P \sim Q$. It will be sufficient to prove $P \sim Q$. From the equivalence of $P$ with $Q$, there is an orthogonal decomposition $Q=\Sigma^{4} E_{i}, E$ are idempotents in $R$ with $P_{i} \sim E_{i}$. Let $Q^{i}$ be the left projection of $\Sigma^{i} E_{j}$, and $Q_{i}=Q^{i}-Q^{i-1}$; as in the argument with the $P_{i}$ 's, the $Q_{i}$ are an orthogonal set of projections adding to $Q$, and $Q_{i} \sim E_{i} \sim P_{i}$, so $Q_{i} \sim P_{i}$. However, going through the same argument as with the $P_{i}$ 's, there exist projections $g_{1}, \ldots, g_{4}$ in $S$ such that $g_{1}, g_{2}$ and $g_{3}, g_{4}$ are orthogonal pairs, and

$$
Q_{1}+Q_{2} \sim\left[\begin{array}{cc}
g_{1}+g_{2} & 0 \\
0 & 0
\end{array}\right] \text { and } Q_{3}+Q_{4} \sim\left[\begin{array}{cc}
0 & 0 \\
0 & g_{3}+g_{4}
\end{array}\right]
$$

It follows from the formulae for ordinary equivalence that $g_{1}+g_{2} \sim f_{1}+f_{2}$ (since $Q_{1}+Q_{2} \sim P_{1}+P_{2}$ ), and similarly with the 3, 4 pairs. As we are working now in $S$, this equivalence is actually a *-equivalence, so

$$
Q_{1}+Q_{2} \dot{\sim}\left[\begin{array}{cc}
g_{1}+g_{2} & 0 \\
0 & 0
\end{array}\right] \dot{\sim}\left[\begin{array}{cc}
f_{1}+f_{2} & 0 \\
0 & 0
\end{array}\right] \dot{\sim} P_{1}+P_{2}
$$

and similarly with $Q_{3}+Q_{4} \sim P_{3}+P_{4}$. Again applying additivity of *-equivalence, we obtain $P \sim Q$, so by 3.1, LP $\sim$ RP holds in $R \stackrel{*}{=} M_{2} S$.

THEOREM 4.6. Let $R$ be a finite Baer ${ }^{*}$ ring satisfying LP $\sim$ RP. If $R$ has sufficiently many projections and has no central abelian projections, or if $R$ has $m$ orthogonal equivalent projections adding to 1 for some $m>3$, then $M_{n} R$ is Baer * for all $n$, and satisfies $\mathrm{LP} \sim \mathrm{RP}$.

Proof. By 3.8 or $3.11, M_{n} R$ is Baer *, and contains all the partial isometries of $M_{n} Q$. Now $Q$ satisfies $\mathrm{LP} \sim \mathrm{RP}$ by 3.3 , and as $Q$ has no central abelian projections (by the usual arguments) $Q=\Pi Q_{i}$, where $Q_{i}$ are various size matrix rings. Each of the $Q_{i}$ satisfy $\mathrm{LP} \sim \mathrm{RP}$ by 4.2 , so by 4.5 , all of the matrix rings over each $Q_{i}$ have this property, and therefore so does $M_{n} Q$. Now $M_{n} Q$ is the maximal ring of quotients of $M_{n} R$, and it is easy to see the right, left projections are the same whether computed in $M_{n} R$ or $M_{n} Q$. As all 
the partial isometries of $M_{n} Q$ lie in $M_{n} R$, all of the *-equivalences can be implemented within $M_{n} R$, and we obtain $M_{n} R$ satisfies $\mathrm{LP} \sim \mathrm{RP}$.

Under the same hypotheses as those of 4.6, it can be shown that such Baer " rings are "*-elementary divisor rings": given a matrix $A$ in $M_{n} R$, there exist unitaries $U, V$ such that $U A V$ is diagonal.

COROLlary 4.7. Let $K$ be a field such that $a^{2}+b^{2}=0$ implies $a=b=0$, for all $a, b$ in $K$. Then $M_{n} K$ is a Baer * ring (with respect to transpose) satisfying LP $\sim \mathrm{RP}$ if and only if for all $a$ in $K$, if $a(1-a)$ is a square in $K$, then $a$ is a square.

Proof. It suffices, in view of 4.5 , to show that $M_{2} K$ satisfies $L P \dot{\sim}$ RP. But this reduces to showing that any projection of trace 1 is *-equivalent to $\left[\begin{array}{c}1 \\ 0 \\ 0\end{array}\right]$; this is a routine computation.

5. The Coordinatization Theorem: An alternate approach. Let $R$ be a Baer * ring where projection lattice is modular ( $R$ is a modular Baer * ring). If the lattice has order $n>4$, then there exists a *-regular ring "coordinatizing" the projection lattice, that is there exists a *-regular ring $C$ such that $L(1, C)$ is ortho-isomorphic to $L(1, R)$. This is a consequence of the famous Coordinatization Theorem of von Neumann [17].

In [2, p. 212], Berberian asks whether given a suitable Baer * ring $R$, does there exist a *-preserving embedding $R \rightarrow C$ such that the projections are the same (equivalently, the map is intimate!). If $R$ is strongly modular, it is easy to see that $Q(R)$ must be *-isomorphic with the 'regular ring' $C$. But there may exist modular Baer * rings of order 4 or more that are not strongly modular-what do we do then? It turns out that we need not worry; all Baer * rings of order 4 or more (as rings, not necessarily according to the order of their projection lattices) that are modular are strongly modular. We prove this by using the methods of proof of the Coordinatization Theorem to get an intimate embedding into $C$. We obtain much more general results on semihereditary rings as well.

Let us revise the notation somewhat. If $R$ is a regular ring we will denote the collection of principal left ideals by $L(R)$. If $R$ is any ring, we will denote the collection of annihilators (i.e., left annihilators) of finite subsets of $M_{n} R$ by $L(n, R)$. We are making this sudden switch from right to left annihilators in order to conform the notation with that in the standard papers dealing with the coordinatization theorem.

A ring is a right (left) p.p. ring if every principal right (left) ideal is projective; equivalently, for all $a$ in $R$, there exists an idempotent $e$ such that $a^{r}=e R$. (Consider the exact sequence, $(0) \rightarrow a^{r} \rightarrow R \rightarrow a R \rightarrow(0)$; this splits.) A ring $R$ is right (left) semihereditary if every finitely generated right 
(left) ideal is projective. If either condition is satisfied on both sides, we drop the right or left qualifiers.

Proposition 5.1. For a ring $R$, the following are equivalent:

(1) for infinitely many $n, M_{n} R$ is left p.p.;

(2) $R$ is left semihereditary;

(3) for any finite subset of $M_{n} R,\left\{a_{i}\right\}$, for any $n$, there exists $e=e^{2}$ in $M_{n} R$ such that

$$
\left\{a_{i}\right\}^{l_{M_{n} R}}=\left(M_{n} R\right) e .
$$

Proof. (1) $\Rightarrow(2)$. Given a finitely generated right ideal, it can be regarded as a principal right ideal of $M_{n} R$ for suitably large $n$, whence is projective.

(2) $\Rightarrow(3)$. Semihereditary is a Morita invariant, so it suffices to prove (3) with $n=1$. Choose a finite subset of $R,\left\{r_{i}\right\}$. We obtain the left exact sequence

$$
(0) \rightarrow\left\{r_{i}\right\}^{\prime} \rightarrow R \stackrel{\beta}{\rightarrow} \oplus R r_{i} \quad \text { where } \beta(1)=\left(r_{1}, r_{2}, \ldots\right) .
$$

Now the image of $\beta$ is a cyclic submodule of a finitely generated free module; thus $\operatorname{Im} \beta$ is projective, so the sequence

$$
(0) \rightarrow\left\{r_{i}\right\}^{\prime} \rightarrow R \rightarrow \operatorname{Im} \beta \rightarrow(0)
$$

splits and thus the term on the left is a direct summand of $R$.

(3) $\Rightarrow(1)$. With $n=1$, we get the condition on $R$ for it to be p.p.

COROLLARY 5.2. If a strongly modular Baer * ring $R$ has all its matrix rings Baer * (or merely even Baer), then the inclusion $R \subset Q(R)$ is a flat epimorphism of rings $(Q(R)$ is the maximal ring of right quotients of $R)$.

Proof. A Baer ring is trivially p.p., so $R$ is semihereditary. Thus the last statement of Theorem 2.3 applies.

Let $A$ be a semihereditary ring. Then $L(n, A)$ is a lattice: the inf (of finite collections of left annihilators of finite sets) is just the intersection, since a finite intersection of left annihilators is the left annihilator of the union of the sets; the sup may be defined as follows:

If $e, f$ are idempotents,

$$
A e \vee A f=((1-e) A \wedge(1-f) A)^{l} ;
$$

equivalently, $A e \bigvee A f=(A e+A f)^{r l}$. (The supremum was defined only for $n=1$, but it is clear, from 5.1, that it makes sense for any $n$.)

Since every element of $L(n, A)$ is generated (as a principal left ideal of $\left.M_{n} A\right)$ by an idempotent, $L(n, A)$ is complemented. Any infinite von Neumann algebra shows $L(n, A)$ is not generally modular. But, if $L(n, A)$ is 
modular for some $n>3$. There is a regular ring $C$ such that $L(C)$, the lattice of principal left ideals of $C$, is lattice isomorphic to $L(n, A)(C$ depends on the choice of $n$, obviously). We construct an embedding from $M_{n} A$ to $C$, that implements this isomorphism, that is, the embedding is intimate.

Given a ring $T=M_{4} A$ such that $L(1, T)=L(4, A)$ is a complemented modular lattice we obtain a ring monomorphism from $A$ to a regular ring $R$ such that $T \rightarrow M_{4} R$ is intimate and $M_{4} R$ coordinatizes $L(1, T)$.

Let $e_{i j}$ denote the $4 \times 4$ matrix over $A$ with 1 in the $(i, j)$ position and 0 elsewhere. Define the left $T$-ideals $b_{0}, b_{1}, b_{2}$ by $b_{i}=T e_{i+1}$ (we abbreviate $e_{j j}$ by $\left.e_{j}\right)$. As the $b_{i}$ are generated by idempotents, $b_{i}$ belong to $L(1, T)$. Define two more principal left ideals $c_{1}=T\left(e_{1}+e_{12}\right), c_{2}=T\left(e_{1}+e_{13}\right)$. Being idempotent-generated, $c_{i}$ belong to $L(1, T)$. This notation is adopted to conform with that in $[11$, p. 90 on]. In $L(1, T)$,

$$
\begin{aligned}
c_{1} \vee c_{2} & =\left(T\left(e_{1}+e_{12}\right)+T\left(e_{1}+e_{13}\right)\right)^{r l} \\
& =\left(\left(1-e_{1}-e_{12}\right) T \cap\left(1-e_{1}-e_{13}\right) T\right)^{l} \\
& =c_{1} \oplus c_{2}
\end{aligned}
$$

as strightforward matrix calculations show. As $b_{1}, b_{2}$ are generated by orthogonal idempotents, $b_{1} \vee b_{2}=b_{1}+b_{2}$. Define $d=\left(c_{1}+c_{2}\right) \cap\left(b_{1}+\right.$ $\left.b_{2}\right) \in L(1, T)$.

If $a, a^{\prime}$ belong to $L(1, T)$, we abbreviate $a \wedge a^{\prime}$ by $a a^{\prime}$.

Let $R$ denote the collection of complements of $b_{1}$ in $b_{1}+b_{0}$ (with respect to $L(1, T)$ ). Multiplication and addition can be defined on $R$ by means of the following formulae [11, p. 91]:

$$
\begin{aligned}
v \boxplus w & =\left\{\left[(v \vee d)\left(b_{0} \vee b_{2}\right)\right] \vee\left[\left(w \vee b_{2}\right)\left(b_{0} \vee d\right)\right]\right\}\left(b_{0} \vee b_{1}\right), \\
v \cdot w & =\left\{\left[\left(v \vee c_{2}\right)\left(b_{1} \vee b_{2}\right)\right] \vee\left[(w \vee d)\left(b_{0} \vee b_{2}\right)\right]\right\}\left(b_{0} \vee b_{1}\right)
\end{aligned}
$$

The proof that $R$ is a regular ring and $M_{4} R$ coordinatizes $L(1, T)$ can be read in the standard texts [17]. We define a function $\beta: A \rightarrow R$ by sending $a \rightarrow^{\beta}\left(-a e_{1}+e_{12}\right)^{r l}$ in $L(1, T)$.

LEMMA 5.3. With the notation of the preceding paragraphs,

$$
\left(-a e_{1}+e_{12}\right)^{r l}=T\left(-a e_{1}+e_{12}\right) \text {. }
$$

Proof. Clearly, $T\left(-a e_{1}+e_{12}\right)=T\left(-a e_{21}+e_{2}\right)$; now $-a e_{21}+e_{2}$ is an idempotent, hence the equation holds.

We may now show that $\beta(a)$ belongs to $R$. Clearly $\beta(a)\left(=T\left(e_{12}-a e_{1}\right)\right)$ has zero intersection with $b_{1}$, and $\beta(a)+b_{1}=b_{1}+b_{0}$. As $b_{1}+b_{0}$ is generated by an idempotent, $\beta(a) \vee b_{1}=b_{1}+b_{0}$, whence $\beta(a)$ belongs to $R$. Now we must verify that $\beta$ is a homomorphism of rings. This is tedious but routine; we only bother checking the additivity of the map and leave the rest. 
Let $v=T\left(-r e_{1}+e_{12}\right), w=T\left(-s e_{1}+e_{12}\right)$. We must show that the $v$ 田 $w$ defined above equals $T\left(-(r+s) e_{1}+e_{12}\right)$. It is not difficult to show $d=\left(b_{1}+b_{2}\right)\left(c_{1}+c_{2}\right)$ equals $T\left(e_{3}-e_{32}\right)$. We first compute $v \vee d$; we must find $\left(T\left(-r e_{1}+e_{12}\right)+T\left(e_{3}-e_{32}\right)\right)^{r l}$, which equals

$$
\left(\left(-r e_{1}+e_{12}\right)^{r} \cap\left(e_{3}-e_{32}\right)^{r}\right)^{l} \text {. }
$$

All the matrices in $\left(-r e_{1}+e_{12}\right)^{r}$ have exactly the form

$$
\left[\begin{array}{cccc}
q & t & u & y \\
r q & r t & r u & r y \\
x & x & x & x \\
x & x & x & x
\end{array}\right]
$$

$q, t, u, y \in A$; the $x$ 's are arbitrary; and all the matrices in $\left(e_{3}-e_{32}\right)^{r}$ have the form

$$
\left[\begin{array}{llll}
x & x & x & x \\
q & t & u & y \\
q & t & u & y \\
x & x & x & x
\end{array}\right]
$$

Intersecting, all the matrices in $\left(-r e_{1}+e_{12}\right)^{r} \cap\left(e_{3}-e_{32}\right)^{r}$ are exactly

$$
\left[\begin{array}{cccc}
q & t & u & y \\
r q & r t & r u & r y \\
r q & r t & r u & r y \\
x & x & x & x
\end{array}\right]
$$

Taking the left annihilator, all the matrices in $v \vee d$ are of the form

$$
\left[\begin{array}{llll}
-(u+t) r & t & u & 0 \\
-(z+y) r & y & z & 0 \\
-(q+p) r & p & q & 0 \\
-(b+a) r & a & b & 0
\end{array}\right], \quad p, a, b, z \in A
$$

Using this form, $v \vee d=T\left(e_{3}-e_{32}\right)+T\left(-r e_{1}+e_{12}\right)$ comes out of a little computation. It also follows from this that

$$
(v \vee d)\left(b_{0}+b_{2}\right)=T\left(-r e_{31}+e_{3}\right)
$$

Now $w=T\left(-s e_{1}+e_{12}\right)=T\left(-s e_{21}+e_{2}\right)$, and $b_{2}=T e_{3}$, so $b_{2}$ and $w$ may be generated by orthogonal idempotents, whence $w \vee b_{2}=w+b_{2}$. It is readily verified that all the matrices in $d+b_{0}$ have exactly the form

$$
\left(\begin{array}{llll}
w & q & -q & 0 \\
a & t & -t & 0 \\
b & u & -u & 0 \\
p & z & -z & 0
\end{array}\right)=T\left(e_{1}+e_{3}-e_{32}\right) .
$$


But $e_{1}+e_{3}-e_{32}$ is an idempotent, so again $d+b_{0}=d \vee b_{0}$. Intersecting $d+b_{0}$ with $w+b_{2}$, we get all the matrices of the form

$$
\left[\begin{array}{llll}
-q s & q & -q & 0 \\
-t s & t & -t & 0 \\
-u s & u & -u & 0 \\
-y s & y & -y & 0
\end{array}\right)
$$

Adding (1) and (2), all the matrices in $(v \vee d)\left(b_{0} \vee b_{2}\right)+\left(w \vee b_{0}\right)\left(d \vee b_{0}\right)$ have exactly the form

$$
\left(\begin{array}{llll}
-q s-p r & q & p-q & 0 \\
-t s-z r & t & z-t & 0 \\
-u s-a r & u & a-u & 0 \\
-y s-b r & y & b-y & 0
\end{array}\right)
$$

Taking the right annihilators of (3), we obtain all matrices of the form

$$
\left[\begin{array}{cccc}
p & q & t & u \\
(s+r) p & (s+r) q & (s+r) t & (s+r) u \\
-r p & -r q & -r t & -r u \\
x & x & x & x
\end{array}\right] .
$$

And taking the left annihilator of (4), we obtain that the matrices in $(v \vee d)\left(b_{0} \vee b_{2}\right) \vee\left(w \vee b_{0}\right)\left(d \vee b_{0}\right)$ are of the form

$$
\left[\begin{array}{llll}
q r-p(s+r) & p & q & 0 \\
u r-t(s+r) & t & u & 0 \\
z r-y(s+r) & y & z & 0 \\
b r-a(s+r) & a & b & 0
\end{array}\right]
$$

Finally intersecting with $b_{0} \vee b_{1}$, we obtain

$$
v \boxplus w=\left(\begin{array}{llll}
-p(s+r) & p & 0 & 0 \\
-t(s+r) & t & 0 & 0 \\
-y(s+r) & y & 0 & 0 \\
-a(s+r) & a & 0 & 0
\end{array}\right)=T\left(-(r+s) e_{12}+e_{1}\right)=\beta(r+s) \text {. }
$$

As $v=\beta(r)$ and $w=\beta(s)$, we have proved the additivity of the map.

Similarly $\beta$ is multiplicative and $\beta(1)$ is the identity of $R$. Now $\beta(r)=0$ precisely if $T\left(-r e_{12}+e_{2}\right)=T e_{2}$; this occurs only if $r=0$. Thus $\beta$ is one-toone. Hence we have proved

Proposition 5.4. Let $A$ be a ring such that $L(4, A)$ is a complemented 
modular lattice. There is an embedding of $A$ into a regular ring $R$ such that $M_{4} R$ coordinatizes $L(4, A)$.

Now we add the slight assumption that every right or left annihilator of a finite set is generated by an idempotent, as occurs for instance if the ring is semihereditary. Now the ring $C$ coordinatizes $L(1, T)$ and the nature of the coordinatization is such that the induced map $T \rightarrow{ }^{M_{4} \beta} C$ assures the map $L(1, T) \rightarrow L(C)$ (the lattice of principal left ideals of $C$, which, of course, equals $L(1, C)$ ) is an isomorphism. Choose $c \in C ; c C \in L(C)$, so there exists $t$ in $T$ with $C \cdot t^{t_{T}}=C c$ (regarding $T$ as a subring of $C$ ). There exists $e=e^{2}$ in $T$ with $t^{l_{T}}=T e$, so $C e=C c$. Thus $T \rightarrow C$ is left intimate. There is an anti-automorphism between left and right annihilators, both in $T$ and in $C$ $(T e \rightarrow(1-e) T$ for example) and all the preceding considerations apply to the opposite rings of $T$ and $C$, so the map is also right intimate.

THEOREM 5.5. Let $T$ be a ring of order 4 or more such that $L(1, T)$ is a complemented modular lattice, and every right or left annihilator of a finite subset of $T$ is generated as a right or left ideal by an idempotent. Then there is an intimate embedding of $T$ into the regular ring coordinatizing $L(1, T)$. In particular, this embedding is an epimorphism of rings.

LEMMA 5.6. Let $T$ be a Baer ${ }^{*}$ ring such that $T=M_{2} S$ (but the involution is not necessarily *-transpose for some involution from $S$ ) and suppose $T$ is intimately embedded in $a{ }^{*}$-regular ring $R$ such that the projection coincide (but the involutions are not required to be the same). Then $T$ is strongly modular, and $R=Q(T)$.

Proof. Suppose $t$ belongs to $T$, and $t^{\prime \tau}=(0)$. Now there exists a projection $p$ in $R$ such that $t^{r_{R}}=p R$; but $p$ belongs to $T$, so $p=0$. Since $L(1, T)$ is complete, it easily follows that $L(1, R)$ is complete, so that $R$ is a complete *-regular ring; by [12, Theorem 2, p. 532], $R$ is directly finite. Since $R$ is regular and $t^{r_{R}}=(0), t$ has a left inverse; by direct finiteness, $t$ has an inverse, so that $t^{l_{R}}=(0)$, and all the more so that $t^{l_{T}}=(0)$. We have thus verified the criterion of 2.9(a) for strong modularity. The last statement is an easy consequence of [17, Theorem 4.1].

THEOREM 5.7. A modular Baer * ring of order 4 or more is strongly modular.

Proof. We have $T=M_{2} S$ for some $S$, and $T$ is intimately embedded in a regular ring $R$ coordinatizing $L(1, T)$, by Theorem 5.5. Now the orthocomplement on $L(1, T), p T \rightarrow(1-p) T$, yields an orthocomplementation on $L(1, R)$, since the inclusion induces an isomorphism of the corresponding lattices (from the definitions of intimate and regular ring!). By [17, Theorem 4.5 , there is an involution on $R$, making $R^{*}$-regular, and fixing the projections of $T$. Since the inclusion is intimate, all the projections of $R$ are already 
in $t$; thus $T$ satisfies all the hypotheses of 5.6.

It should be possible to prove 5.7 without having to resort to the tedious and highly computational use of the Coordinatization Theorem.

COROLlaRY 5.8. Let $R$ be a finite Baer * ring satisfying SR, and suppose $R$ has $m$ orthogonal equivalent idempotents adding to 1 , for some $m>3$. Then $R$ is strongly modular, and thus satisfies $\mathrm{LP} \sim \mathrm{RP}$, and all its matrix rings are strongly modular and Baer *.

Proof. By [22, Theorem 2.1], $R$ satisfies the parallelogram law with respect to *-equivalence; by the proof of [13, Theorem 67$], L(1, R)$ is modular, and so by $5.7, R$ is strongly modular. Also by [22, Theorem 2.1$]$, GC holds; now in a ring with SR, equivalence implies *-equivalence [13, Theorem 27], and it easily follows that $R$ is an $m \times m^{*}$-matrix ring. Thus 3.10 applies.

6. Examples. We construct two mildly interesting examples. The first is a strongly modular factor of type II that does not satisfy any of the usual set of axioms (it does not satisfy $L P \sim R P, S R$ or possess sufficiently many projections), and all its matrix rings are Baer *. (In fact it is *-isomorphic to all its matrix rings, as is to be expected with type II factors.) The second example is a directly finite Baer * factor (regrettably not of type II) of any fixed order, having GC, that is not strongly modular (see 3.10).

Example 1. Begin with the field of rational numbers, $\mathbf{Q}$, and form the polynomial ring $\mathbf{Q}[x]$, and its quotient field $K=\mathbf{Q}(x)$. Then with the transpose involution, $M_{n} K$ is a ${ }^{*}$-regular ring, and of course a Baer *-ring. Now form the local domain obtained by inverting all the elements in $Q[x]$ that are relatively prime to $x$; call this domain $D$. Explicitly,

$$
D=\{f / g \in K \mid f, g \in \mathbf{Q}[x],(f, g) \mathbf{Q}[x]=\mathbf{Q}[x] \text {, and } g(0) \neq 0\} .
$$

We will show that for all $n, M_{n} D$ is a Baer * ring with respect to transpose (compare with $\mathrm{Q}[x]: M_{2} \mathrm{Q}[x]$ is not a Baer * ring, although it is a Baer ring). It is sufficient (and also necessary, but this is not relevant) to show $M_{n} D$ contains all the projections of $M_{n} K$.

Obviously $D /(x)$ is just $Q$. Choose a matrix $A$ in $M_{n} K$ such that $A^{2}=A=$ $A^{t}\left({ }^{t}=\right.$ transpose). As $D$ is a local principal ideal domain, there exists an integer $m$ such that $x^{m} A \in M_{n} D$, but $x^{m} A \notin M_{n}(x D)$. Set $x^{m} A=B$. Then $B$ satisfies: $B^{2}=x^{m} B$, and $B=B^{t}$. Suppose $m>0$. Then in $D /(x)$ (i.e. Q) the image of $B, \bar{B}$, satisfies $\bar{B}^{2}=0$, and $\bar{B}=\bar{B}^{t}$. But a symmetric matrix over the rationals is only nilpotent if it is 0 , which would contradict $x^{m} A \notin M_{n}(x D)$. Thus $m<0$, and so $A \in M_{n} D$. So $M_{n} D$ contains all the projections of $M_{n} K$ and it is easy to see this makes $M_{n} D$ into a Baer * ring.

We may now form the ring $R=\operatorname{Lim} M_{2^{n}} K$, obtained by mapping the matrices diagonally $\left(A \rightarrow\left[\begin{array}{ll}A & 0 \\ 0\end{array}\right]\right)$. Then $R$ is a ${ }^{*}$-regular ring, and possesses a 
unique rank function $N$. By [9, Corollary 7] the completion of $R, \bar{R}$ with respect to this rank function is a regular Baer ${ }^{*}$ factor of type $\mathrm{II}_{f}$, with centre $K$. Now consider the subring of $R, T=\operatorname{Lim} M_{2^{n}} D$, and complete it at the rank function induced by that on $R$; call the completion $\bar{T}$. $\bar{T}$ is obviously closed under the involution; we show it contains all the projections of $\bar{R}$, and is therefore a Baer * ring.

Lemma 6.1. Let $R$ be $a^{*}$-regular ring with a rank function $N$. Let $\bar{R}$ denote the completion of $R$ at $N$. Then the involution of $R$ extends uniquely to $\bar{R}$, making $\bar{R}$ into a Baer * ring. Every projection of $\bar{R}$ is a limit of projections from $R$.

Proof. All but the last statement is in [9, Proposition 1]. Choose a projection $p$ in $\bar{R}$, and $\varepsilon>0$. There exists $b$ in $R$ with $N(p-b)<\varepsilon / 20$. Define $a=\left(b+b^{*}\right) / 2$. As $N(r)=N\left(r^{*}\right)$ for all $r$ in $\bar{R}$ (cf. the proof the result referred to above in [9]), we have $a=a^{*}$ and $N(p-a)<\varepsilon / 10$. Now $a^{2}-a=a-p+p(p-a)+(p-a) a$, so $N\left(a^{2}-a\right) \leqslant 3 N(p-a)<$ $3 \varepsilon / 10$. Since $R$ is *-regular, there exists a unique $t$ satisfying $a t=t a$, at $a=a$, tat $=t$ (apply [12, Lemma 4] and observe that $a$ is symmetric); it follows that $t=t^{*}$. Set $q=a t$; then $q$ is a projection, and as

$$
q-t=a^{2} t^{2}-a t^{2}=\left(a^{2}-a\right) t^{2},
$$

we obtain $N(q-t)<3 \varepsilon / 10$. But, $q-t^{2}=(q-t) q+t(q-t)$, whence $N\left(q-t^{2}\right)<6 \varepsilon / 10$. But $t^{2}-t=q-t-\left(q-t^{2}\right)$; thus $N\left(t^{2}-t\right)<9 \varepsilon / 10$. Further, $q-a=\left(t^{2}-t\right) a^{2}$, so $N(q-a)<9 \varepsilon / 10$. Finally, $N(p-q)<$ $N(p-a)+N(a-q)<10 \varepsilon / 10=\varepsilon$. Thus $p$ may be approximated by projections from $R$.

As $T$ contains all the projections of $R$, by the lemma, $\bar{T}$ contains all the projections of $\bar{R}$, and as $\bar{T}$ is invariant under the involution, $\bar{T}$ is a Baer *-subring of $\bar{R}$, in particular $\bar{T}$ is a Baer * ring.

Now the ideal of $\bar{T}$ generated by $x$ contains no nonzero projections. To see this, observe that if $p=x r(r$ in $\bar{T})$, then $p^{2}=p$ yields $(x r-1) r=0$ (as $x$ is central and not a zero divisor). Now choose a matrix $A$ in $M_{2^{n}} D . D$ is integrally closed in $K$, so all the eigenvalues for $A$ that lie in $K$ are already in $D$. Thus $1 / x$ (in $K$ ) is not an eigenvalue for $A$, and so $A-(1 / x) I$ is invertible, so $N((A-1 / x) A)=N(A)$ for the rank function $N$. Thus for all $t$ in $\bar{T}$ we have $N((x t-1) t)=N(t)$, which yields $r=0$, so $p=0$. Thus $\bar{t}$ does not have sufficiently many projections.

Next it is routine to verify that 2 has no 'square root' in the sense of 3.7(b), so LP $\sim$ RP fails in $\bar{T}$ (and also $\bar{R}$ ), and neither does $1+x^{2}$, so that SR fails. $T$ itself is isomorphic to matrix rings over itself, so $\bar{T}$ is also so isomorphic. $\bar{T}$ is intimately embedded in the Baer * ring $\bar{R}$, which has arbitrarily large order, 
so $\bar{T}$ is strongly modular. That it is a factor is trivial, as is the fact that it is of type II.

$\bar{R}$ is not obtainable from $\bar{T}$ by inverting central elements; it may be possible to obtain $\bar{R}$ by inverting a multiplicatively closed collection of suitable non-zero-divisors; but it appears unlikely that $\bar{T}$ is an order in $\bar{R}$.

This construction raises the interesting problem of determining which domains have their matrix rings Baer *. Necessary and sufficient conditions for an arbitrary commutative ring to have all its matrix rings Baer * are determined in a forthcoming paper by the author.

EXAMPLE 2. Directly finite, not modular, of large order.

Let $\mathbf{R}$ denote the real numbers. Set $T=\mathbf{R}[[x, y]$, that is the power series ring over the reals in two noncommuting variables. The involution on $T$ is that generated by $x \mapsto x, y \mapsto y$ (so $x y$ is sent to $y x$ ). As is well known, $T$ is embeddable in a division ring, so $M_{n} T$, being subrings of artinian rings, have no infinite chains of right or left annihilators, i.e., all annihilators are annihilators of finite subsets. But $T$ is a free ideal ring, in particular $T$ is semihereditary, so that by $5.1, M_{n} T$ is a Baer ring. Now the Jacobson radical of $M_{n} T$ is just the two-sided ideal generated by the $T$-ideal, [[x,y]]; with *-transpose as involution \#, it readily follows that for any $A$ in $M_{n} T$, $1+A A^{\#}$ is invertible. By [13, Theorem 26], $M_{n} T$ is Baer *.

$T$ is a local domain, so $M_{n} T$ is directly finite for all $n$.

Were $M_{n} T$ modular for some $n>3$, it would be strongly modular, and so $T$ itself would have to be strongly modular; this is equivalent to $T$ being an Ore domain, which, of course, $T$ is not $(x T \cap y T=(0))$. Thus $M_{n} T$ is a Baer * ring of order $n$ that is directly finite, but not modular, if $n>3$ (this can probably be reduced to $n>1$ ).

It is not easy to see that GC holds in $M_{n} T$ (cf. Corollary 3.10 and the comment following it).

With a little work, this example can probably be made into a type II factor (it is now of type $\mathrm{I}_{n}$ ).

7. Problems. 1. Characterize flat epimorphisms that are intimate. For example, if $R$ is an order in $S$, and $S$ is regular, the inclusion is intimate.

2. If $R \rightarrow S$ is intimate, $S$ is regular, and $S$ is of sufficiently large (matrix) order (perhaps 2 is large enough), is ${ }_{R} S$ flat? Is $M_{2} S$ an intimate extension of $M_{2} R$ ?

3. If $T$ is a type II finite $A W^{*}$ or $W^{*}$ algebra, then its 'regular ring' is a flat epimorphic extension (Corollary 5.2). Problem. Find an application for this.

4. One obstacle implicit in $\$ 5$ is the order assumption on the modular Baer * ring $T$. If $L(1, T)$ has order $n$ (as a lattice), does $T$ have order $n$ (as a ring)? The converse is trivial. In order to construct the map $\beta$ of $\S 5$, it appears to be necessary that the ring, and not just the lattice, have sufficiently high order. 
This problem would be resolved if one knew that perspectivity of projections implies equivalence.

\section{BIBLIOGRAPHY}

1. S. K. Berberian, $N \times N$ matrices over a finite $A W^{*}$-algebra, Amer. J. Math. 80 (1958), 37-44. MR 20 \# 4790.

3. J. E. Burke, On the property (PU) of *-regular rings, Canad. Math. Bull. 19 (1976), 21-38.

4. V. C. Cateforis, Flat regular quotient rings, Trans. Amer. Math. Soc. 138 (1969), 241-249. MR 39 \#259.

5. K. R. Goodearl, D. Handelman and J. Lawrence, Strongly prime and completely torsion free rings, Carleton Math. Lecture Notes, no. 109, Carleton Univ., Ottawa, Canada, 1974.

6. I. Hafner, The regular ring and the maximal ring of quotients of a finite Baer *-ring, Michigan Math. J. 21 (1974), 153-160.

7. D. Handelman, Strongly prime, simple self-injective, and completely torsion-free rings, $\mathrm{Ph}$. $\mathrm{D}$. Dissertation, McGill Univ.,1975.

8. __ Perspectivity and cancellation in regular rings, J. Algebra (to appear).

9. Completions of rank rings, Canad. Math. Bull. (to appear).

10. J. M. Howie and J. R. Isbell, Epimorphisms and dominions. II, J. Algebra 6 (1967), 7-21. MR 35 \# 105b.

11. B. Jónsson, Representations of complemented modular lattices, Trans. Amer. Math. Soc. 97 (1960), 64-94. MR 22 \# 10932.

12. I. Kaplansky, Any orthocomplemented complete modular lattice is a continuous geometry, Ann. of Math. (2) 61 (1955), 524-541. MR 19, 524.

13. __ Rings of operators, Benjamin, New York, 1968. MR 39 \#6092.

14. J. Lambek, Lectures on rings and modules, Blaisdell, Waltham, Mass., 1966. MR 34 \#5857.

15. __ Localization at epimorphisms and quasi-injectives, J. Algebra 38 (1976), 163-181.

16. S. Maeda and S. S. Holland, Jr., Equivalence of projections in Baer * rings, J. Algebra 39 (1976), 150-159. MR 53 \#8121.

17. J. von Neumann, Continuous geometry, Princeton Univ. Press, Princeton, N. J., 1960. MR $22 \# 10931$.

18. N. Prijatelj and I. Vidav, On special *-regular rings, Michigan Math. J. 18 (1971), 213-221. MR 44 \#257.

19. E. Pyle, The regular ring and the maximal ring of quotients of a finite Baer *-ring, Trans. Amer. Math. Soc. 203 (1975), 201-213. MR 51 \#593.

20. J.-E. Roos, Sur l'anneau maximal de fractions des $A W^{*}$-algèbres et des anneaux de Baer, C. R. Acad. Sci. Paris Sér. A-B 266 (1968), A120-A123. MR 39 \#6093.

21. B. T. Stenström, Rings and modules of quotients, Lecture Notes in Math., vol. 237, Springer-Verlag, Berlin and New York, 1971. MR 48 \# 4010.

22. Y. Utumi, On the continuity and self-injectivity of a complete regular ring, Canad. J. Math. 18 (1966), 404-412. MR 36 \#6457.

23. On continuous rings and self-injective rings, Trans. Amer. Math. Soc. 118 (1965), 158-173. MR 30 \# 4793.

24. On rings of which any one-sided quotient rings are two-sided, Proc. Amer. Math. Soc. 14 (1963), 141-147. MR 26 \# 137.

25. I. Vidav, On some * regular rings, Acad. Serbe Sci. Publ. Inst. Math. 13 (1959), 73-80. MR 23 \# A4029.

Department of Mathematics, University of Utah, Salt Lake City, Utah 84112

Mathematisches Institut, Justus Liebig-Universträt, Giessen, West Germany

Current address: Department of Mathemtatics, University of Ottawa, Ottawa, Ontario, Canada K1N 6N5 